\title{
Direct Aminoalkylation of Arenes and Hetarenes via Ni- Catalyzed Negishi Cross-Coupling Reactions
}

\author{
Laurin Melzig, Andrey Gavryushin and Paul Knochel* \\ Depa $25^{\circ} \mathrm{C}$ ment Chemie und Biochemie, Ludwig-Maximilians-Universität, \\ Butenandtstrasse 5-13, 81377, Munich (Germany). \\ Paul.Knochel@cup.uni-muenchen.de
}

\section{Supporting information}

Experimental procedures, analytical and spectroscopy data for final products (30 pages). 


\section{General considerations.}

All reactions were carried out under an argon atmosphere in dried glassware. THF was continuously refluxed and freshly distilled from sodium benzophenone ketyl under nitrogen. Yields refer to isolated yields of compounds estimated to be $>97 \%$ pure as determined by ${ }^{1} \mathrm{H}-\mathrm{NMR}\left(25^{\circ} \mathrm{C}\right)$ and capillary GC analysis.

\section{General Procedure 1: Preparation of aminoalkylmagnesium chlorides by insertion of} magnesium.

Magnesium turnings (1.2 equiv.) and $\mathrm{LiCl}$ (2.0 equiv.) were placed in an argon flushed threenecked flask and dried for $10-20 \mathrm{~min}$ at $130{ }^{\circ} \mathrm{C}$ under vacuum $(1 \mathrm{mbar})$. The flask was refilled with argon and then cooled under argon. After the addition of THF ( $500 \mathrm{~mL}$ per $1 \mathrm{~mol}$ of the alkyl chloride) and DIBAL-H (20\% in hexane, $3 \mathrm{~mol} \%)$, the alkyl chloride, dissolved in THF $(500 \mathrm{~mL}$ per $1 \mathrm{~mol})$ was added dropwise so that the mixture was gently boiling. Then, the reaction mixture was refluxed for another $2 \mathrm{~h}$. The solution was titrated prior to use at room temperature against a solution of iodine in THF. A concentration of $0.3 \mathrm{M}$ to $0.8 \mathrm{M}$ in THF was obtained.

\section{General Procedure 2: Cross-coupling of the aminoalkylmagnesium chlorides with aryl electrophiles using $\mathrm{Ni}(\text { acac })_{2}$ and DPE-Phos.}

A dry Schlenk flask, equipped with a magnetic stirring bar, was charged with a solution of $\mathrm{ZnBr}_{2}(450 \mathrm{mg}, 2 \mathrm{mmol})$ in THF $(1 \mathrm{~mL})$ and $N$-methylpyrrolidone $(0.3 \mathrm{~mL})$. The mixture was stirred at room temperature for $15 \mathrm{~min}$. The aminoalkylmagnesium chloride $(1.2 \mathrm{mmol})$ was added and the mixture was stirred at room temperature for $15 \mathrm{~min}$. The electrophile (1.0 mmol), DPE-Phos (27 mg, $5 \mathrm{~mol} \%)$ and $\mathrm{Ni}(\mathrm{acac})_{2}(9 \mathrm{mg}, 2.5 \mathrm{~mol} \%)$ dissolved in THF (1 $\mathrm{mL}$ ) were added and the mixture was stirred at room temperature until the complete consumption of the electrophile was observed by GC analysis.

The reaction mixture was quenched with sat. aq. $\mathrm{K}_{2} \mathrm{CO}_{3}$ solution $(20 \mathrm{~mL})$, extracted with diethyl ether $(3 \times 20 \mathrm{~mL})$. The combined organic layers were extracted with $2 \mathrm{~N} \mathrm{HCl}(2 \times 20$ $\mathrm{mL})$ and water $(20 \mathrm{~mL})$. The acidic aqueous layer was basified with sat. $\mathrm{K}_{2} \mathrm{CO}_{3}$ and extracted with diethyl ether $(3 \times 20 \mathrm{~mL})$, the extract dried over anhydrous $\mathrm{Na}_{2} \mathrm{SO}_{4}$. After filtration, the solvent was evaporated in vacuo and purified by column chromatography, if necessary.

Synthesis of 3-(dimethylamino)propylmagnesium chloride.<smiles>CN(C)CCCN(C)Cl</smiles>

(3-Chloropropyl)dimethylamine (12.16 g, $100 \mathrm{mmol})$ was reacted with $\mathrm{Mg}$ turnings (2.88 $\mathrm{g}$, $120 \mathrm{mmol})$ and $\mathrm{LiCl}(8.48 \mathrm{~g}, 200 \mathrm{mmol})$ according to TP 1 . The solution was titrated prior to use at room temperature against a solution of iodine in THF. A concentration of $0.80 \mathrm{M}$ in THF was obtained, $82 \%$ yield. 
Synthesis of 2-(1-methylpiperidin-2-yl)ethylmagnesium chloride.

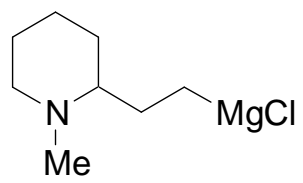

2-(2-Chloroethyl)-1-methylpiperidine $(16.17 \mathrm{~g}, 100 \mathrm{mmol})$ was reacted with $\mathrm{Mg}$ turnings $(2.88 \mathrm{~g}, 120 \mathrm{mmol})$ and $\mathrm{LiCl}(8.48 \mathrm{~g}, 200 \mathrm{mmol})$ according to TP 1. The solution was titrated prior to use at room temperature against a solution of iodine in THF. A concentration of 0.71 $\mathrm{M}$ in THF was obtained, $66 \%$ yield.

Synthesis of (1-methylpiperidin-3-yl)methylmagnesium chloride.<smiles>CN1CCCC(COCCCl)C1</smiles>

3-(Chloromethyl)-1-methylpiperidine (14.77 $\mathrm{g}, 100 \mathrm{mmol})$ was reacted with $\mathrm{Mg}$ turnings $(2.88 \mathrm{~g}, 120 \mathrm{mmol})$ and $\mathrm{LiCl}(8.48 \mathrm{~g}, 200 \mathrm{mmol})$ according to TP $\mathbf{1}$. The solution was titrated prior to use at room temperature against a solution of iodine in THF. A concentration of 0.50 $\mathrm{M}$ in THF was obtained, $77 \%$ yield.

Synthesis of (1-methylpiperidin-4-yl)magnesium chloride.<smiles>CN1CCC(CCl)CC1</smiles>

4-Chloro-1-methylpiperidine (13.36 g, $100 \mathrm{mmol}$ ) was reacted with $\mathrm{Mg}$ turnings (2.88 g, 120 $\mathrm{mmol})$ and $\mathrm{LiCl}(8.48 \mathrm{~g}, 200 \mathrm{mmol})$ according to TP 1. The solution was titrated prior to use at room temperature against a solution of iodine in THF. A concentration of $0.62 \mathrm{M}$ in THF was obtained, $56 \%$ yield.

\section{Synthesis of 3-chloro-8-methyl-8-azabicyclo[3.2.1]octane.}

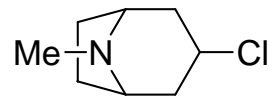

Solution of tropine $(28.3 \mathrm{~g} 200 \mathrm{mmol})$ in $\mathrm{CHCl}_{3}(250 \mathrm{~mL})$ was cooled down to $-30^{\circ} \mathrm{C}$. Thionyl chloride (47.6 g, $400 \mathrm{mmol}$ ) was added slowly and the solution was stirred at reflux temperature for $3 \mathrm{~h}$ and then additional $16 \mathrm{~h}$ at room temperature. The solution was carefully mixed with $6 \mathrm{~N} \mathrm{NaOH}$, aqueous phase extracted with $\mathrm{CH}_{2} \mathrm{Cl}_{2} / \mathrm{Et}_{2} \mathrm{O}$ (1:1) and washed with sat. aq. $\mathrm{K}_{2} \mathrm{CO}_{3}$ solution. The $\mathrm{K}_{2} \mathrm{CO}_{3}$ solution was extracted with $\mathrm{CH}_{2} \mathrm{Cl}_{2}$ three times and the combined organic layers were dried over anhydrous $\mathrm{Na}_{2} \mathrm{SO}_{4}$. After removal of the solvent in vacuo, the crude product was purified by fractionated distillation in vacuo, which furnished 3chloro-8-methyl-8-azabicyclo[3.2.1]octane (14.9 g, $93.0 \mathrm{mmol}, 47 \%)$ as a colorless oil. 
B.p.: $80^{\circ} \mathrm{C}(12 \mathrm{mbar})$.

${ }^{\mathbf{1}} \mathbf{H}-\mathbf{N M R}\left(\mathrm{CDCl}_{3}, 300 \mathrm{MHz}, 25^{\circ} \mathrm{C}\right): \delta[\mathrm{ppm}]=4.18-4.06(\mathrm{~m}, 1 \mathrm{H}), 3.18-3.16(\mathrm{~m}, 2 \mathrm{H}), 2.31(\mathrm{~s}$, $3 \mathrm{H}), 2.07-1.90(\mathrm{~m}, 6 \mathrm{H}), 1.58-1.51(\mathrm{~m}, 2 \mathrm{H})$.

${ }^{13} \mathbf{C}-\mathbf{N M R}\left(\mathrm{CDCl}_{3}, 75 \mathrm{MHz}, 25^{\circ} \mathrm{C}\right): \delta[\mathrm{ppm}]=61.4,53.3,41.1,38.7,26.7$.

MS (EI, $70 \mathrm{eV}), \mathrm{m} / \mathrm{z}(\%): 159$ (7) [M+], 124 (100), 96 (11), 94 (3), 82 (8), 67 (7).

HRMS: calculated for $\mathrm{C}_{8} \mathrm{H}_{14} \mathrm{CIN} 159.0815$, found 159.0813 .

IR (ATR): 0 [ $\left.\mathrm{cm}^{-1}\right]=2936$ (vs), 2878 (s), $2798(\mathrm{~m}), 1449$ (m), 1336 (s), 1301 (m), 1238 (s), 1129 (w), 1042 (m), 1027 (s), 866 (m), 832 (m), 762 (s), 640 (m)

The analytical data match those from the literature ${ }^{1}$.

Synthesis of 8-methyl-8-azabicyclo[3.2.1]octyl-3-magnesium chloride.

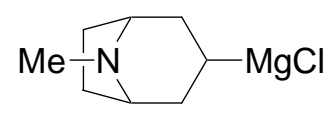

3-Chloro-8-methyl-8-azabicyclo[3.2.1]octane (15.97 g, $100 \mathrm{mmol}$ ) was reacted with $\mathrm{Mg}$ turnings $(2.88 \mathrm{~g}, 120 \mathrm{mmol})$ and $\mathrm{LiCl}(8.48 \mathrm{~g}, 200 \mathrm{mmol})$ according to TP $\mathbf{1}$. The fresh solution was titrated prior to use at room temperature against a solution of iodine in THF. A concentration of $0.3 \mathrm{M}$ in THF was obtained, $35 \%$ yield.

\section{[3-(3-Methoxy-phenyl)propyl]-dimethylamine (3a)}

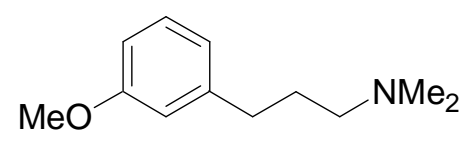

3-Bromoanisole (187 mg, $1.0 \mathrm{mmol})$ was reacted with 3-(dimethylamino)propylmagnesium chloride $(0.8 \mathrm{M}$ in THF, $1.5 \mathrm{~mL}, 1.2 \mathrm{mmol})$ at $25^{\circ} \mathrm{C}$ for $3 \mathrm{~h}$, according to TP 2. Aqueous workup furnished [3-(3-methoxy-phenyl)-propyl]-dimethyl-amine (188 mg, $0.97 \mathrm{mmol}, 97 \%$ yield) as a yellow oil.

${ }^{\mathbf{1}} \mathbf{H}-\mathbf{N M R}\left(\mathrm{CDCl}_{3}, 300 \mathrm{MHz}, 25^{\circ} \mathrm{C}\right): \delta[\mathrm{ppm}]=7.21-7.15(\mathrm{~m}, 1 \mathrm{H}), 6.79-6.70(\mathrm{~m}, 3 \mathrm{H}), 3.78(\mathrm{~s}$, $3 \mathrm{H}), 2.63-2.58(\mathrm{t}, \mathrm{J}=7.8 \mathrm{~Hz}, 2 \mathrm{H}), 2.31-2.26(\mathrm{t}, \mathrm{J}=7.3 \mathrm{~Hz}, 2 \mathrm{H}), 2.21(\mathrm{~s}, 6 \mathrm{H}), 1.83-1.73(\mathrm{~m}$, 2H).

${ }^{13} \mathrm{C}-\mathrm{NMR}\left(\mathrm{CDCl}_{3}, 75 \mathrm{MHz}, 25^{\circ} \mathrm{C}\right): \delta[\mathrm{ppm}]=159.9,144.2,129.5,121.0,114.4,111.3,59.5$, 55.4, 45.7, 34.0, 29.6 .

MS (EI, $70 \mathrm{eV}), \mathrm{m} / \mathrm{z}(\%): 193(43)\left[\mathrm{M}^{+}\right], 122(11), 121(8), 91$ (7), 59 (4), 58 (100).

HRMS: calculated for $\mathrm{C}_{12} \mathrm{H}_{19} \mathrm{NO} 193.1467$, found 193.1466.

\footnotetext{
${ }^{1}$ S. Archer, M. R. Bell, T. R. Lewis, J. W. Schulenberg, M. J. Unser, J. Am. Chem. Soc. 1957, 79, 6337-6338.
} 
IR (ATR): 0 [ $\left[\mathrm{cm}^{-1}\right]=3382$ (vs), 3014 (m), 2942 (s), 2836 (m), 1600 (s), 1584 (s), 1486 (vs), 1466 (s), 1438 (s), 1258 (vs), 1154 (s), 1038 (s), 916 (m), 886 (w), 784 (m), 696 (m).

\section{$N, N$-Dimethyl-(3-quinolin-2-yl-propyl)amine (3b)}

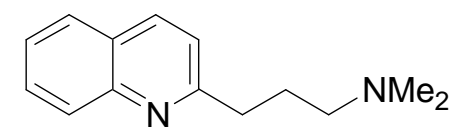

2-Chloroquinoline (164 mg, $1.0 \mathrm{mmol}$ ) was reacted with (3-(dimethylamino)propyl magnesium chloride $(0.8 \mathrm{M}$ in THF, $1.5 \mathrm{~mL}, 1.2 \mathrm{mmol})$ at $25^{\circ} \mathrm{C}$ for $3 \mathrm{~h}$, according to TP 2 . Aequous workup furnished $\mathrm{N}, \mathrm{N}$-dimethyl-(3-quinolin-2-yl-propyl)amine (189 $\mathrm{mg}, 0.88$ mmol, $88 \%$ yield) as a brownish oil.

${ }^{1} \mathbf{H}-\mathbf{N M R}\left(\mathrm{CDCl}_{3}, 300 \mathrm{MHz}, 25^{\circ} \mathrm{C}\right): \delta[\mathrm{ppm}]=7.97-7.92(\mathrm{~m}, 2 \mathrm{H}), 7.66-7.63(\mathrm{~m}, 1 \mathrm{H}), 7.60-$ $7.54(\mathrm{~m}, 1 \mathrm{H}), 7.38-7.33(\mathrm{~m}, 1 \mathrm{H}), 7.19(\mathrm{~d}, \mathrm{~J}=8.4 \mathrm{~Hz}, 1 \mathrm{H}), 2.91(\mathrm{t}, \mathrm{J}=7.8 \mathrm{~Hz}, 2 \mathrm{H}), 2.30(\mathrm{t}, \mathrm{J}=$ $8.0 \mathrm{~Hz}, 2 \mathrm{H}), 2.15(\mathrm{~s}, 6 \mathrm{H}), 1.98-1.88(\mathrm{~m}, 2 \mathrm{H})$.

${ }^{13} \mathrm{C}-\mathrm{NMR}\left(\mathrm{CDCl}_{3}, 75 \mathrm{MHz}, 25^{\circ} \mathrm{C}\right): \delta[\mathrm{ppm}]=162.5,148.1,136.4,129.5,129.0,127.7$, $126.9,125.9,121.6,59.4,45.6,37.1,27.9$.

MS (EI, $70 \mathrm{eV}), \mathrm{m} / \mathrm{z}(\%): 170(6)\left[\mathrm{M}^{+}\right], 143$ (100), 128 (17), 115 (7), 72 (19), 58 (80)

HRMS: calculated for $\mathrm{C}_{14} \mathrm{H}_{18} \mathrm{~N}_{2} 214.1470$, found 214.1477 .

IR (ATR): $\ddot{v}\left[\mathrm{~cm}^{-1}\right]=2940(\mathrm{~m}), 2858(\mathrm{w}), 2814(\mathrm{~m}), 2764(\mathrm{~m}), 1618(\mathrm{~m}), 1600(\mathrm{~s}), 1562(\mathrm{~m})$, 1504 (s), 1462 (m), 1426 (s), 1140 (m), 1040 (m), 826 (vs), 748 (vs), 618 (m)

\section{$N, N$-Dimethyl-3-(quinoxalin-6-yl)propan-1-amine (3c)}

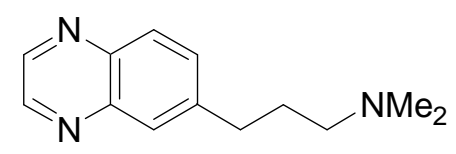

6-Bromoquinoxaline (209 $\mathrm{mg}, \quad 1.0 \mathrm{mmol}$ ) was reacted with (3-(dimethylamino) propylmagnesium chloride $(0.8 \mathrm{M}$ in THF, $1.5 \mathrm{~mL}, 1.2 \mathrm{mmol})$ at $25^{\circ} \mathrm{C}$ for $1 \mathrm{~h}$, according to TP 2. Aqueous workup furnished $N, N$-dimethyl-3-(quinoxalin-6-yl)propan-1-amine (211 mg, $0.98 \mathrm{mmol}, 98 \%$ yield) as an orange-brown oil.

${ }^{1} \mathbf{H}-\mathbf{N M R}\left(\mathrm{CDCl}_{3}, 600 \mathrm{MHz}, 25^{\circ} \mathrm{C}\right): \delta[\mathrm{ppm}]=8.64-8.62(\mathrm{~m}, 2 \mathrm{H})$, 7.87-7.86 (m, $\left.1 \mathrm{H}\right)$, 7.75$7.74(\mathrm{~m}, 1 \mathrm{H}), 7.48-7.47(\mathrm{~m}, 1 \mathrm{H}), 2.72(\mathrm{t}, \mathrm{J}=7.7 \mathrm{~Hz}, 2 \mathrm{H}), 2.17(\mathrm{t}, \mathrm{J}=7.3 \mathrm{~Hz}, 2 \mathrm{H}), 2.07$ (s, $6 \mathrm{H}), 1.81-1.71(\mathrm{~m}, 2 \mathrm{H})$.

${ }^{13} \mathbf{C}-\mathbf{N M R}\left(\mathrm{CDCl}_{3}, 150 \mathrm{MHz}, 25^{\circ} \mathrm{C}\right): \delta[\mathrm{ppm}]=145.0,144.3,143.2,141.8,131.7,129.3$, 127.8, 59.1, 45.6, 33.7, 29.0.

MS (EI, $70 \mathrm{eV}), \mathrm{m} / \mathrm{z}(\%): 215$ (6) [M+ $], 169$ (1), 142 (4), 71 (9), 58 (100). 
HRMS: calculated for $\mathrm{C}_{13} \mathrm{H}_{17} \mathrm{~N}_{3}$ 215.1422, found 215.1422.

IR (ATR): $\left.0 \mathrm{~cm}^{-1}\right]=2940(\mathrm{~m}), 2856(\mathrm{~m}), 2814(\mathrm{~m}), 2764(\mathrm{~m}), 1620(\mathrm{~m}), 1498(\mathrm{~s}), 1452(\mathrm{~s})$, 1368 (m), 1132 (m), 1024 (vs), 958 (s), 896 (m), 864 (s), 830 (s), 664 (m)

3-(6-Methoxypyridine-2-yl)- $N, N$-dimethylpropan-1-amine (3d)<smiles>COc1cccc(CCCN(C)C)n1</smiles>

2-Chloro-6-methoxypyridine (144 mg, $1.0 \mathrm{mmol}$ ) was reacted with (3-(dimethylamino)propyl magnesium chloride $(0.8 \mathrm{M}$ in THF, $1.5 \mathrm{~mL}, 1.2 \mathrm{mmol})$ at $25{ }^{\circ} \mathrm{C}$ for $1 \mathrm{~h}$, according to $\mathbf{T P} \mathbf{2}$. Aqueous workup furnished 2-(3-dimethylaminopropyl)-6-methoxypyridine (164 mg, 0.85 mmol, $85 \%$ yield) as a yellow-orange oil.

${ }^{\mathbf{1}} \mathbf{H}-\mathbf{N M R}\left(\mathrm{CDCl}_{3}, 600 \mathrm{MHz}, 25^{\circ} \mathrm{C}\right): \delta[\mathrm{ppm}]=7.37-7.34(\mathrm{~m}, 1 \mathrm{H}), 6.61(\mathrm{~d}, \mathrm{~J}=7.3 \mathrm{~Hz}, 1 \mathrm{H})$, $6.44(\mathrm{~d}, \mathrm{~J}=8.4 \mathrm{~Hz}, 1 \mathrm{H}), 3.82(\mathrm{~s}, 3 \mathrm{H}), 2.62(\mathrm{t}, \mathrm{J}=7.7 \mathrm{~Hz}, 2 \mathrm{H}), 2.29(\mathrm{t}, \mathrm{J}=7.5 \mathrm{~Hz}, 2 \mathrm{H}), 2.14$ (s, 6H), 1.86-1.80 (m, 2H).

${ }^{13} \mathbf{C}-\mathbf{N M R}\left(\mathrm{CDCl}_{3}, 150 \mathrm{MHz}, 25^{\circ} \mathrm{C}\right): \delta[\mathrm{ppm}]=163.8,159.8,138.8,115.2,107.5,59.5,53.2$, 45.6, 35.7, 27.4 .

MS (EI, $70 \mathrm{eV}), \mathrm{m} / \mathrm{z}(\%): 195$ (8) [M+H'] 136 (6), 124 (13), 123 (100), 72 (26), 58 (56).

HRMS: calculated for $\mathrm{C}_{11} \mathrm{H}_{19} \mathrm{~N}_{2} \mathrm{O}^{+}$195.1492, found 195.1517.

IR (ATR): $\hat{0}\left[\mathrm{~cm}^{-1}\right]=2946$ (m), 2856 (w), 2814 (w), 2764 (w), 1598 (s), 1578 (vs), 1462 (vs), 1440 (s), 1412 (s), 1288 (s), 1262 (m), 1150 (m), 1030 (s), 986 (m), 798 (s), 738 (m)

1-[4-(3-Dimethylamino-propyl)-phenyl]-pentan-1-one (3e)<smiles>CN(C)CCCc1ccc(C(=O)Br)cc1</smiles>

1-(4-Bromo-phenyl)-pentan-1-one (241 mg, $1.0 \mathrm{mmol}$ ) was reacted with (3-(dimethylamino) propylmagnesium chloride $(0.8 \mathrm{M}$ in THF, $1.5 \mathrm{~mL}, 1.2 \mathrm{mmol})$ at $25{ }^{\circ} \mathrm{C}$ for $1 \mathrm{~h}$, according to TP 2. Aqueous workup furnished 1-[4-(3-dimethylamino-propyl)-phenyl]-pentan-1-one (223 $\mathrm{mg}, 0.90 \mathrm{mmol}, 90 \%$ yield) as a yellow-orange oil.

${ }^{1} \mathbf{H}$-NMR $\left(\mathrm{CDCl}_{3}, 300 \mathrm{MHz}, 25^{\circ} \mathrm{C}\right): \delta[\mathrm{ppm}]=7.84(\mathrm{~d}, \mathrm{~J}=8.4 \mathrm{~Hz}, 2 \mathrm{H}), 7.23(\mathrm{~d}, \mathrm{~J}=8.4 \mathrm{~Hz}$, 2H), $2.90(\mathrm{t}, \mathrm{J}=7.5 \mathrm{~Hz}, 2 \mathrm{H}), 2.66(\mathrm{t}, \mathrm{J}=7.7 \mathrm{~Hz}, 2 \mathrm{H}), 2.25(\mathrm{t}, \mathrm{J}=7.5 \mathrm{~Hz}, 2 \mathrm{H}), 2.18(\mathrm{~s}, 6 \mathrm{H})$, 1.81-1.81 (m, 2H), 1.73-1.63 (m, 2H), 1.43-1.31 (m, 2H), 0.91 (t, J = 7.3 Hz, 3H).

${ }^{13} \mathrm{C}-\mathrm{NMR}\left(\mathrm{CDCl}_{3}, 75 \mathrm{MHz}, 25^{\circ} \mathrm{C}\right): \delta[\mathrm{ppm}]=200.3,148.1,135.2,128.8,128.4,59.2,45.7$, $38.4,33.8,29.3,26.8,22.7,14.2$.

MS (EI, $70 \mathrm{eV}), \mathrm{m} / \mathrm{z}(\%): 247$ (3) [M+], 145 (3), 115 (2), 91 (1), 58 (100). 
HRMS: calculated for $\mathrm{C}_{16} \mathrm{H}_{25} \mathrm{NO} 247.1936$, found 247.1913 .

IR (ATR): 0 [ $\left[\mathrm{cm}^{-1}\right]=2936$ (m), 2860 (m), $2814(\mathrm{w}), 2764$ (m), 1680 (vs), 1606 (s), 1460 (m), $1412(\mathrm{~m}), 1266$ (m), 1214 (m), $1180(\mathrm{~s}), 1012$ (m), 968 (m), $846(\mathrm{~m})$

\section{3-(2-(1-Methylpiperidin-2-yl)ethyl)benzonitrile (3f)}<smiles>CN1CCCCC1CCc1cccc(C#N)c1</smiles>

3-Bromobenzonitrile (182 $\mathrm{mg}, 1.0 \mathrm{mmol})$ was reacted with 2-(1-methylpiperidin-2yl)ethylmagnesium chloride $(0.8 \mathrm{M}$ in THF, $1.5 \mathrm{~mL}, 1.2 \mathrm{mmol})$ at $25^{\circ} \mathrm{C}$ for $0.5 \mathrm{~h}$, according to TP 2. Aqueous workup furnished 3-(2-(1-methylpiperidin-2-yl)ethyl)benzonitrile (220 mg, $0.96 \mathrm{mmol}, 96 \%$ yield) as a yellow-orange oil.

${ }^{1} \mathbf{H}-\mathbf{N M R}\left(\mathrm{CDCl}_{3}, 300 \mathrm{MHz}, 25^{\circ} \mathrm{C}\right): \delta[\mathrm{ppm}]=7.35-7.33(\mathrm{~m}, 2 \mathrm{H}), 7.31-7.30(\mathrm{~m}, 1 \mathrm{H}), 7.28-$ $7.25(\mathrm{~m}, 1 \mathrm{H}), 2.75-2.58(\mathrm{~m}, 2 \mathrm{H}), 2.54-2.43(\mathrm{~m}, 1 \mathrm{H}), 2.15(\mathrm{~s}, 3 \mathrm{H}), 2.01-1.12(\mathrm{~m}, 10 \mathrm{H}$.

${ }^{13} \mathrm{C}-\mathrm{NMR}\left(\mathrm{CDCl}_{3}, 75 \mathrm{MHz}, 25^{\circ} \mathrm{C}\right): \delta[\mathrm{ppm}]=144.4,133.1,131.9,129.6,129.3,119.1$, 112.5, 63.2, 57.3, 43.0, 34.6, 30.8, 30.7, 25.9, 24.6.

MS (EI, $70 \mathrm{eV}), \mathrm{m} / \mathrm{z}(\%): 228$ (2) [M+], 171 (1), 115 (3), 98 (100), 70 (7).

HRMS: calculated for $\mathrm{C}_{15} \mathrm{H}_{20} \mathrm{~N}_{2} 228.1626$, found 228.1623.

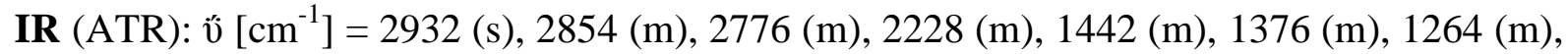
$1124(\mathrm{~m}), 1032(\mathrm{~m}), 886(\mathrm{w}), 796(\mathrm{~s}), 690(\mathrm{vs})$

\section{2-Methoxy-6-(2-(1-methylpiperidin-2-yl)ethyl)pyridine (3g)}<smiles>COc1cccc(CCC2CCCCN2C)n1</smiles>

2-Chloro-6-methoxy-pyridine (144 mg, $1.0 \mathrm{mmol})$ was reacted with 2-(1-methylpiperidin-2yl)ethylmagnesium chloride $(0.8 \mathrm{M}$ in THF, $1.5 \mathrm{~mL}, 1.2 \mathrm{mmol})$ at $25{ }^{\circ} \mathrm{C}$ for $0.5 \mathrm{~h}$, according to TP 2. Aqueous workup furnished 2-methoxy-6-(2-(1-methylpiperidin-2-yl)ethyl)pyridine (219 mg, $0.94 \mathrm{mmol}, 94 \%$ yield) as a yellow-orange oil.

${ }^{\mathbf{1}} \mathbf{H}$-NMR $\left(\mathrm{CDCl}_{3}, 300 \mathrm{MHz}, 25^{\circ} \mathrm{C}\right): \delta[\mathrm{ppm}]=7.37-7.30(\mathrm{~m}, 1 \mathrm{H}), 6.59(\mathrm{dd}, \mathrm{J}=7.3 \mathrm{~Hz}, 2.9$ $\mathrm{Hz}, 1 \mathrm{H}), 6.42(\mathrm{dd}, \mathrm{J}=8.2 \mathrm{~Hz}, 2.9 \mathrm{~Hz}, 1 \mathrm{H}), 3.80$ (s, 3H), 2.77-2.64 (m, 2H), 2.60-2.49 (m, $1 \mathrm{H}), 2.19(\mathrm{~s}, 3 \mathrm{H}), 2.02-1.92(\mathrm{~m}, 2 \mathrm{H}), 1.86-1.62(\mathrm{~m}, 4 \mathrm{H}), 1.49-1.48(\mathrm{~m}, 2 \mathrm{H}), 1.34-1.13(\mathrm{~m}$, $2 \mathrm{H})$. 
${ }^{13} \mathrm{C}-\mathrm{NMR}\left(\mathrm{CDCl}_{3}, 75 \mathrm{MHz}, 25^{\circ} \mathrm{C}\right): \delta[\mathrm{ppm}]=163.8,160.4,138.7,115.1,107.5,63.5,57.4$, 53.2, 43.2, 33.4, 32.5, 30.9, 26.1, 24.6.

MS (EI, $70 \mathrm{eV}), \mathrm{m} / \mathrm{z}(\%): 234$ (1) [M+], 136 (2), 122 (83), 112 (60), 98 (100), 70 (7).

HRMS: calculated for $\mathrm{C}_{14} \mathrm{H}_{22} \mathrm{~N}_{2} \mathrm{O} 234.1732$, found 234.1710 .

IR (ATR): $\ddot{0}\left[\mathrm{~cm}^{-1}\right]=2932(\mathrm{~m}), 2854(\mathrm{~m}), 2776$ (m), 1598 (s), 1578 (vs), 1464 (vs), 1440 (s), 1412 (s), 1286 (s), 1264 (s), 1146 (m), 1030 (vs), 798 (s), 732 (m)

\section{2-(2-(1-Methylpiperidin-2-yl)ethyl)quinoline (3h)}<smiles>CN1CCCCC1CCc1ccc2ccccc2n1</smiles>

2-Chloroquinoline (164 mg, $1.0 \mathrm{mmol})$ was reacted with 2-(1-methylpiperidin-2yl)ethylmagnesium chloride $(0.8 \mathrm{M}$ in THF, $1.5 \mathrm{~mL}, 1.2 \mathrm{mmol})$ at $25^{\circ} \mathrm{C}$ for $1 \mathrm{~h}$, according to TP 2. Aqueous workup furnished 2-(2-(1-methylpiperidin-2-yl)ethyl)quinoline (241 mg, 0.95 mmol, $95 \%$ yield) as an orange-brown oil.

${ }^{1} \mathbf{H}-\mathbf{N M R}\left(\mathrm{CDCl}_{3}, 300 \mathrm{MHz}, 25^{\circ} \mathrm{C}\right): \delta[\mathrm{ppm}]=7.99-7.94(\mathrm{~m}, 2 \mathrm{H}), 7.67(\mathrm{~d}, \mathrm{~J}=7.5 \mathrm{~Hz}, 1 \mathrm{H})$, 7.62-7.56 (m, 1H), $7.38(\mathrm{t}, \mathrm{J}=7.5 \mathrm{~Hz}, 1 \mathrm{H}), 7.20(\mathrm{t}, \mathrm{J}=8.4 \mathrm{~Hz}, 1 \mathrm{H}), 3.05-2.95(\mathrm{~m}, 1 \mathrm{H}), 2.91-$ $2.75(\mathrm{~m}, 2 \mathrm{H}), 2.23(\mathrm{~s}, 3 \mathrm{H}), 2.13-1.79(\mathrm{~m}, 4 \mathrm{H}), 1.75-1.64(\mathrm{~m}, 2 \mathrm{H}), 1.56-1.48(\mathrm{~m}, 2 \mathrm{H}), 1.42-$ $1.12(\mathrm{~m}, 2 \mathrm{H})$.

${ }^{13} \mathrm{C}-\mathrm{NMR}\left(\mathrm{CDCl}_{3}, 75 \mathrm{MHz}, 25^{\circ} \mathrm{C}\right): \delta[\mathrm{ppm}]=163.1,148.1,136.4,129.5,129.1,127.7$, $126.9,125.8,121.5,63.6,57.4,43.3,35.0,33.0,30.9,26.1,24.6$.

MS (EI, $70 \mathrm{eV}), \mathrm{m} / \mathrm{z}(\%): 253$ (1) [M-H'] 168 (3), 155 (3), 143 (100), 112 (58), 98 (45), 70 (3).

HRMS: calculated for $\mathrm{C}_{17} \mathrm{H}_{21} \mathrm{~N}_{2}{ }^{+} 253.1699$, found 253.1701 .

IR (ATR): $\dddot{0}\left[\mathrm{~cm}^{-1}\right]=2930(\mathrm{~s}), 2854(\mathrm{~m}), 2776(\mathrm{~m}), 1618(\mathrm{~m}), 1600(\mathrm{~s}), 1502(\mathrm{~s}), 1442(\mathrm{~m})$, 1426 (m), $1374(\mathrm{~m}), 1138(\mathrm{~m}), 1118(\mathrm{~m}), 1032$ (m), 824 (vs), 756 (s), 618 (m)

\section{6-[2-(1-Methyl-piperidin-2-yl)-ethyl]-quinoxaline (3i)}<smiles>CN1CCCCC1CCc1ccc2nccnc2c1</smiles>

6-Bromoquinoxaline (209 $\mathrm{mg}, 1.0 \mathrm{mmol})$ was reacted with 2-(1-methylpiperidin-2yl)ethylmagnesium chloride $(0.8 \mathrm{M}$ in THF, $1.5 \mathrm{~mL}, 1.2 \mathrm{mmol})$ at $25^{\circ} \mathrm{C}$ for $3 \mathrm{~h}$, according to TP 2. Aqueous workup and purification by flash chromatography $\left(\mathrm{CH}_{2} \mathrm{Cl}_{2} /\right.$ methanol, 10:1) furnished 6-[2-(1-methyl-piperidin-2-yl)-ethyl]-quinoxaline (232 mg, $0.91 \mathrm{mmol}, 91 \%$ yield) as an orange-brown oil. 
${ }^{1} \mathbf{H}-\mathbf{N M R}\left(\mathrm{CDCl}_{3}, 600 \mathrm{MHz}, 25^{\circ} \mathrm{C}\right): \delta[\mathrm{ppm}]=8.74-8.72(\mathrm{~m}, 2 \mathrm{H}), 7.96(\mathrm{~d}, \mathrm{~J}=8.6 \mathrm{~Hz}, 1 \mathrm{H})$, $7.83(\mathrm{~s}, 1 \mathrm{H}), 7.57(\mathrm{t}, \mathrm{J}=8.4 \mathrm{~Hz}, 1 \mathrm{H}), 7.20(\mathrm{t}, \mathrm{J}=8.4 \mathrm{~Hz}, 1 \mathrm{H}), 2.94-2.89(\mathrm{~m}, 1 \mathrm{H}), 2.85-2.83$ $(\mathrm{m}, 1 \mathrm{H}), 2.79-2.74(\mathrm{~m}, 1 \mathrm{H}), 2.27(\mathrm{~s}, 3 \mathrm{H}), 2.10-2.06(\mathrm{~m}, 1 \mathrm{H}), 1.99-1.94(\mathrm{~m}, 2 \mathrm{H}), 1.86-1.81(\mathrm{~m}$, $1 \mathrm{H}), 1.71-1.69(\mathrm{~m}, 2 \mathrm{H}), 1.56-1.55(\mathrm{~m}, 2 \mathrm{H}), 1.46-1.40(\mathrm{~m}, 1 \mathrm{H}), 1.27-1.17(\mathrm{~m}, 1 \mathrm{H})$.

${ }^{13} \mathrm{C}-\mathrm{NMR}\left(\mathrm{CDCl}_{3}, 150 \mathrm{MHz}, 25^{\circ} \mathrm{C}\right): \delta[\mathrm{ppm}]=145.4,145.1,144.4,143.3,141.9,131.7$, 129.4, 127.7, 63.4, 57.2, 42.9, 34,2. 31,4. 30.6, 25.7, 24.4 .

MS (EI, $70 \mathrm{eV}), \mathrm{m} / \mathrm{z}(\%): 255$ (2) [M+], 157 (1), 142 (4), 111 (5), 98 (100), 70 (6).

HRMS: calculated for $\mathrm{C}_{16} \mathrm{H}_{21} \mathrm{~N}_{3} 255.1735$, found 255.1733 .

IR (ATR): $\hat{0}\left[\mathrm{~cm}^{-1}\right]=2930$ (vs), 2854 (m), 2776 (s), 1620 (w), 1498 (m), 1450 (m), 1366 (m), $1266(\mathrm{w}), 1132$ (m), $1024(\mathrm{~m}), 890(\mathrm{~m}), 864(\mathrm{~m}), 826(\mathrm{w}), 730(\mathrm{w})$.

\section{2-Methoxy-6-(1-methyl-piperidin-3-ylmethyl)-pyridine (3j)}<smiles>COc1cccc(CC2CCCN(C)C2)n1</smiles>

2-Chloro-6-methoxypyridine (144 mg, $1.0 \mathrm{mmol}$ ) was reacted with (1-methylpiperidin-3yl)methylmagnesium chloride $(0.5 \mathrm{M}$ in THF, $2.4 \mathrm{~mL}, 1.2 \mathrm{mmol})$ at $25^{\circ} \mathrm{C}$ for $20 \mathrm{~h}$, according to TP 2. Aqueous workup furnished furnished 2-methoxy-6-(1-methyl-piperidin-3-ylmethyl)pyridine (198 mg, $0.90 \mathrm{mmol}, 90 \%$ yield) as a yellow-orange oil.

${ }^{\mathbf{1}} \mathbf{H}$-NMR $\left(\mathrm{CDCl}_{3}, 300 \mathrm{MHz}, 25^{\circ} \mathrm{C}\right): \delta[\mathrm{ppm}]=7.38-7.33(\mathrm{~m}, 1 \mathrm{H}), 6.58(\mathrm{~d}, \mathrm{~J}=7.1 \mathrm{~Hz}, 1 \mathrm{H})$, $6.45(\mathrm{~d}, \mathrm{~J}=8.4 \mathrm{~Hz}, 1 \mathrm{H}), 3.82(\mathrm{~s}, 3 \mathrm{H}), 2.68(\mathrm{~d}, \mathrm{~J}=11.0 \mathrm{~Hz}, 2 \mathrm{H}), 2.49(\mathrm{~d}, \mathrm{~J}=7.1 \mathrm{~Hz}, 2 \mathrm{H}), 2.14$ (s, 3H), 2.12-2.00 (m, 1H), 1.83-1.75 (m, 1H), 1.64-1.42 (m, 4H), 0.93-0.80 (m, 1H).

${ }^{13} \mathrm{C}-\mathrm{NMR}\left(\mathrm{CDCl}_{3}, 75 \mathrm{MHz}, 25^{\circ} \mathrm{C}\right): \delta[\mathrm{ppm}]=163.8,158.5,138.6,116.0,107.7,62.3,56.5$, 53.3, 46.9, 43.1, 36.7, 30.6, 25.7.

MS (EI, 70 eV), m/z (\%): 219 (8) [M-H'] 123 (100), 98 (26), 70 (2), 58 (2), 43 (4).

HRMS: calculated for $\mathrm{C}_{13} \mathrm{H}_{20} \mathrm{~N}_{2} \mathrm{O} 220.1576$, found 220.1613 .

IR (ATR): $\dddot{0}\left[\mathrm{~cm}^{-1}\right]=2932$ (m), 2848 (w), 2774 (m), 1598 (s), 1578 (s), 1464 (vs), 1438 (s), 1414 (s), 1302 (s), 1262 (s), 1146 (m), 1032 (s), 798 (s), 780 (s), 746 (m)

\section{5-(1-Methyl-piperidin-3-ylmethyl)-nicotinic acid ethyl ester (3k)}<smiles>CCOc1cncc(CC2CCCN(C)C2)c1</smiles>

5-Bromopyridine-3-carboxylic acid ethyl ester $(230 \mathrm{mg}, 1.0 \mathrm{mmol})$ was reacted with (1methylpiperidin-3-yl)methylmagnesium chloride $(0.5 \mathrm{M}$ in THF, $2.4 \mathrm{~mL}, 1.2 \mathrm{mmol})$ at $25^{\circ} \mathrm{C}$ 
for $20 \mathrm{~h}$, according to TP 2. Aqueous workup and purification by flash chromatography $\left(\mathrm{CH}_{2} \mathrm{Cl}_{2}\right.$ /methanol, 20:1) furnished 5-(1-methyl-piperidin-3-ylmethyl)-nicotinic acid ethyl ester (204 mg, $0.78 \mathrm{mmol}, 78 \%$ yield) as a yellow-orange oil.

${ }^{1} \mathbf{H}-\mathbf{N M R}\left(\mathrm{CDCl}_{3}, 300 \mathrm{MHz}, 25^{\circ} \mathrm{C}\right): \delta[\mathrm{ppm}]=8.91(\mathrm{~s}, 1 \mathrm{H}), 8.42(\mathrm{~s}, 1 \mathrm{H}), 7.94(\mathrm{~s}, 1 \mathrm{H}), 4.26$ $(\mathrm{q}, \mathrm{J}=7.3 \mathrm{~Hz}, 2 \mathrm{H}), 2.70-2.61(\mathrm{~m}, 2 \mathrm{H}), 2.45(\mathrm{~d}, \mathrm{~J}=7.1 \mathrm{~Hz}, 2 \mathrm{H}), 2.13(\mathrm{~s}, 3 \mathrm{H}), 1.88-1.81(\mathrm{~m}$, 2H), 1.66-1.45 (m, 4H), $1.27(\mathrm{t}, \mathrm{J}=7.3 \mathrm{~Hz}, 3 \mathrm{H}), 0.89-0.78(\mathrm{~m}, 1 \mathrm{H})$.

${ }^{13} \mathrm{C}-\mathrm{NMR}\left(\mathrm{CDCl}_{3}, 75 \mathrm{MHz}, 25^{\circ} \mathrm{C}\right): \delta[\mathrm{ppm}]=.5,154.0,148.8,137.3,135.4,126.1,61.5$, $61.4,56.0,46.4,37.7,37.5,29.9,24.9,14.4$.

MS (EI, $70 \mathrm{eV}), \mathrm{m} / \mathrm{z}(\%): 262$ (44) [M+], 233 (11), 217 (13), 111 (14), 97 (100), 71 (17), 58 (32), 43 (26).

HRMS: calculated for $\mathrm{C}_{15} \mathrm{H}_{22} \mathrm{~N}_{2} \mathrm{O}_{2} 262.1681$, found 262.1670 .

IR (ATR): $\ddot{v}\left[\mathrm{~cm}^{-1}\right]=2932(\mathrm{~m}), 2850(\mathrm{w}), 2776(\mathrm{w}), 1720(\mathrm{vs}), 1446(\mathrm{~m}), 1368(\mathrm{~m}), 1288$ (vs), 1206 (s), 1178 (m), 1160 (m), 1104 (s), 1024 (s), 864 (w), 764 (s), 710 (m), 676 (w).

\section{4-(3-Methoxy-phenyl)-1-methyl-piperidine (3I)}

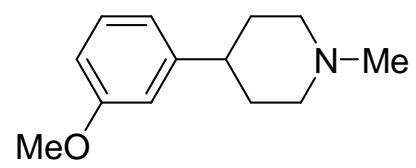

1-Bromo-3-methoxybenzene $(187 \mathrm{mg}, 1.0 \mathrm{mmol})$ was reacted with (1-methylpiperidin-4yl)magnesium chloride $(0.6 \mathrm{M}$ in THF, $2.0 \mathrm{~mL}, 1.2 \mathrm{mmol})$ at $25^{\circ} \mathrm{C}$ for $6 \mathrm{~h}$, according to $\mathbf{T P}$ 2. Aequous workup furnished 4-(3-methoxy-phenyl)-1-methyl-piperidine (189 mg, 0.92 mmol, $92 \%$ yield) as a yellow oil.

${ }^{1} \mathbf{H}-\mathbf{N M R}\left(\mathrm{CDCl}_{3}, 300 \mathrm{MHz}, 25^{\circ} \mathrm{C}\right): \delta[\mathrm{ppm}]=7.23-7.18(\mathrm{~m}, 1 \mathrm{H}), 6.82-6.71(\mathrm{~m}, 3 \mathrm{H}), 3.79(\mathrm{~s}$, $3 \mathrm{H}), 2.94-2.87(\mathrm{~m}, 2 \mathrm{H}), 2.55-2.43(\mathrm{~m}, 1 \mathrm{H}), 2.24(\mathrm{~s}, 3 \mathrm{H}), 2.03-1.92(\mathrm{~m}, 2 \mathrm{H}), 1.90-1.82(\mathrm{~m}$, $4 \mathrm{H})$

${ }^{13} \mathbf{C}-\mathrm{NMR}\left(\mathrm{CDCl}_{3}, 75 \mathrm{MHz}, 25^{\circ} \mathrm{C}\right): \delta[\mathrm{ppm}]=160.2,144.3,129.8,121.1,114.7,111.2,59.4$, $55.2,45.7,42.9,32.3$.

MS (EI, $70 \mathrm{eV}), \mathrm{m} / \mathrm{z}(\%): 205$ (4) [M+], 134 (13), 121 (9), 97 (100), 83 (29), 70 (69), 58 (22), $43(47)$

HRMS: calculated for $\mathrm{C}_{13} \mathrm{H}_{19} \mathrm{NO} 205,1467$, found 205.1474 .

IR (ATR): $\ddot{v}\left[\mathrm{~cm}^{-1}\right]=3371$ (vs), 3006 (m), 2910 (s), 1633 (m), 1559 (s), 1479 (vs), 1495 (s), 1233 (s), 1201 (m), 1152 (s), 1070 (s), 1011 (m), 905 (w), 856 (m), 784 (m), 696 (m). 


\section{1-[4-(1-Methyl-piperidin-4-yl)-phenyl]-pentan-1-one (3m)}

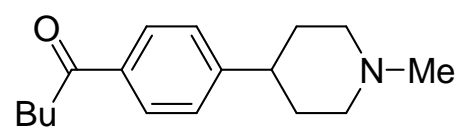

1-(4-Bromo-phenyl)-pentan-1-one $(241 \mathrm{mg}, 1.0 \mathrm{mmol})$ was reacted with (1-methylpiperidin4-yl)magnesium chloride ( $0.6 \mathrm{M}$ in THF, $2.0 \mathrm{~mL}, 1.2 \mathrm{mmol})$ at $25^{\circ} \mathrm{C}$ for $2 \mathrm{~h}$, according to $\mathbf{T P}$ 2. Aqueous workup furnished 1-[4-(1-methyl-piperidin-4-yl)-phenyl]-pentan-1-one (218 mg, $0.84 \mathrm{mmol}, 84 \%$ yield) as an orange solid.

Mp.: $37-38^{\circ} \mathrm{C}$.

${ }^{1} \mathbf{H}-\mathbf{N M R}\left(\mathrm{CDCl}_{3}, 300 \mathrm{MHz}, 25^{\circ} \mathrm{C}\right): \delta[\mathrm{ppm}]=7.80(\mathrm{~d}, \mathrm{~J}=8.2 \mathrm{~Hz}, 2 \mathrm{H}), 7.20(\mathrm{~d}, \mathrm{~J}=8.2 \mathrm{~Hz}$, $2 \mathrm{H}), 2.89-2.81(\mathrm{~m}, 4 \mathrm{H}), 2.49-2.38(\mathrm{~m}, 1 \mathrm{H}), 2.22(\mathrm{~s}, 3 \mathrm{H}), 2.00-1.91(\mathrm{~m}, 2 \mathrm{H}), 1.75-1.68(\mathrm{~m}$, $4 \mathrm{H}), 1.66(\mathrm{~m}, 2 \mathrm{H}), 1.37-1.24(\mathrm{~m}, 2 \mathrm{H}), 0.84(\mathrm{t}, \mathrm{J}=7.3 \mathrm{~Hz}, 3 \mathrm{H})$.

${ }^{13} \mathbf{C}$-NMR $\left(\mathrm{CDCl}_{3}, 75 \mathrm{MHz}, 25^{\circ} \mathrm{C}\right): \delta[\mathrm{ppm}]=200.3,151.9,135.4,128.5,127.2,56.3,46.6$, $42.3,38.4,33.4,26.8,22.7,14.1$.

MS (EI, $70 \mathrm{eV}), \mathrm{m} / \mathrm{z}(\%): 259$ (90) [M+], 258 (100), 202 (4), 97 (15), 83 (5), 70 (13), 43 (3).

HRMS: calculated for $\mathrm{C}_{17} \mathrm{H}_{25} \mathrm{NO} 259.1936$, found 260.1947 .

IR (ATR): 0 $\left[\mathrm{cm}^{-1}\right]=2934$ (m), $2872(\mathrm{~m}), 2778(\mathrm{~m}), 1678$ (vs), 1606 (s), 1464 (m), 1444 (m), 1378 (m), 1278 (m), 1212 (m), 1182 (m), 1134 (m), 992 (m), 976 (m), $844(\mathrm{~m}), 764(\mathrm{~m})$

\section{4-(1-Methyl-piperidin-4-yl)-benzonitrile (3n)}

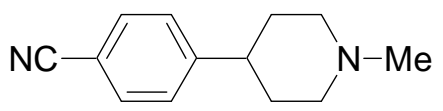

4-Cyanophenyl triflate $(251 \mathrm{mg}, 1.0 \mathrm{mmol})$ was reacted with (1-methylpiperidin-4yl)magnesium chloride (0.6 M in THF, $2.0 \mathrm{~mL}, 1.2 \mathrm{mmol})$ at $25^{\circ} \mathrm{C}$ for $8 \mathrm{~h}$, according to TP2. Aqueous workup furnished 4-(1-methyl-piperidin-4-yl)-benzonitrile (189 mg, $0.95 \mathrm{mmol}, 95$ $\%$ yield) as a yellow-orange solid.

Мp.: $70-72^{\circ} \mathrm{C}$.

${ }^{\mathbf{1}} \mathbf{H}$-NMR $\left(\mathrm{CDCl}_{3}, 300 \mathrm{MHz}, 25^{\circ} \mathrm{C}\right): \delta[\mathrm{ppm}]=7.51(\mathrm{~d}, \mathrm{~J}=8.2 \mathrm{~Hz}, 2 \mathrm{H}), 7.26(\mathrm{~d}, \mathrm{~J}=8.2 \mathrm{~Hz}$, $2 \mathrm{H}), 2.93-2.90(\mathrm{~m}, 2 \mathrm{H}), 2.53-2.42(\mathrm{~m}, 1 \mathrm{H}), 2.25(\mathrm{~s}, 3 \mathrm{H}), 2.03-1.95(\mathrm{~m}, 2 \mathrm{H}), 1.77-1.64(\mathrm{~m}$, $4 \mathrm{H})$. 
${ }^{13} \mathbf{C}-\mathbf{N M R}\left(\mathrm{CDCl}_{3}, 75 \mathrm{MHz}, 25^{\circ} \mathrm{C}\right): \delta[\mathrm{ppm}]=152.0,132.5,127.9,119.2,110.2,56.2,46.6$, 42.4, 33.3.

MS (EI, $70 \mathrm{eV)}, \mathrm{m} / \mathrm{z}(\%): 200$ (87) [M+], 199 (100), 128 (4), 115 (7), 97 (12), 83 (12), 70 (27), 42 (19).

HRMS: calculated for $\mathrm{C}_{13} \mathrm{H}_{16} \mathrm{~N}_{2} 200.1313$, found 200.1289 .

IR (ATR): $\ddot{0}\left[\mathrm{~cm}^{-1}\right]=2940(\mathrm{~s}), 2834(\mathrm{~m}), 2786(\mathrm{~s}), 2738(\mathrm{~m}), 2222(\mathrm{~s}), 1606(\mathrm{~m}), 1508(\mathrm{~m})$, 1468 (m), 1446 (s), 1416 (m), 1378 (m), 1276 (s), 1128 (m), 1080 (m), 1064 (m), 994 (s), 836 (vs), 768 (s)

exo-2-Fluoro-4-(8-methyl-8-aza-bicyclo[3.2.1]oct-3-yl)benzonitrile (3o)

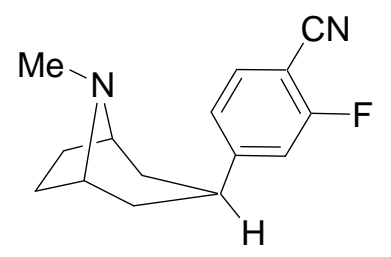

4-Bromo-2-fluorobenzonitrile (200 mg, $1.0 \mathrm{mmol})$ was reacted with 8-methyl-8-azabicyclo[3.2.1] octyl-3-magnesium chloride $(0.3 \mathrm{M}$ in THF, $4.0 \mathrm{~mL}, 1.2 \mathrm{mmol})$ at $25^{\circ} \mathrm{C}$ for 30 $\mathrm{h}$, according to TP2. Aqueous workup and purification by flash chromatography $\left(\mathrm{CH}_{2} \mathrm{Cl}_{2}\right.$ /methanol, 20:1) furnished exo-2-fluoro-4-(8-methyl-8-aza-bicyclo[3.2.1]oct-3-yl)benzonitrile (190 mg, $0.78 \mathrm{mmol}, 78 \%$ yield) as an orange-red oil.

${ }^{1} \mathbf{H}-\mathbf{N M R}\left(\mathrm{CDCl}_{3}, 600 \mathrm{MHz}, 25^{\circ} \mathrm{C}\right): \delta[\mathrm{ppm}]=7.40-7.37(\mathrm{~m}, 1 \mathrm{H}), 7.02(\mathrm{~d}, \mathrm{~J}=8.2 \mathrm{~Hz}, 1 \mathrm{H})$, $6.98(\mathrm{~d}, 1 \mathrm{H}, \mathrm{J}=10.4 \mathrm{~Hz}, 1 \mathrm{H}), 3.14-3.10(\mathrm{~m}, 2 \mathrm{H}), 2.82-2.76(\mathrm{~m}, 1 \mathrm{H}), 2.18(\mathrm{~s}, 3 \mathrm{H}), 2.10-1.97$ $(\mathrm{m}, 4 \mathrm{H}), 1.55-1.50(\mathrm{~m}, 4 \mathrm{H})$.

${ }^{13} \mathbf{C}-\mathbf{N M R}\left(\mathrm{CDCl}_{3}, 150 \mathrm{MHz}, 25^{\circ} \mathrm{C}\right): \delta[\mathrm{ppm}]=163.4,155.3,133.4,124.0,115.3,114.3$, $98.9,61.5,40.7,38.8,35.3,26.1$.

MS (EI, 70 eV), m/z (\%): 244 (51) [M+'], 215 (38), 201 (8), 134 (8), 96 (32), 83 (100), 57 (6), 42 (37).

HRMS: calculated for $\mathrm{C}_{15} \mathrm{H}_{17} \mathrm{FN}_{2} 244.1376$, found 244.1375.

IR (ATR): $\ddot{v}\left[\mathrm{~cm}^{-1}\right]=2958$ (s), 2920 (vs), 2848 (m), 2228 (m), 1618 (vs), 1566 (m), 1502 (s), 1450 (s), 1430 (s), 1352 (m), 1256 (m), 1110 (s), 968 (m), 816 (s), 764 (s).

exo-[4-(8-Methyl-8-aza-bicyclo[3.2.1]oct-3-yl)-phenyl]acetonitrile (3p)

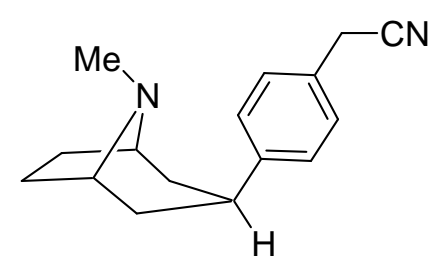

(4-Bromo-phenyl)acetonitrile (196 $\mathrm{mg}, 1.0 \mathrm{mmol})$ was reacted with 8-methyl-8-azabicyclo[3.2.1] octyl-3-magnesium chloride (0.3 M in THF, $4.0 \mathrm{~mL}, 1.2 \mathrm{mmol})$ at $25^{\circ} \mathrm{C}$ for 72 $\mathrm{h}$, according to TP2. Aqueous workup and purification by flash chromatography $\left(\mathrm{CH}_{2} \mathrm{Cl}_{2}\right.$ 
/methanol, 20:1) furnished exo-[4-(8-methyl-8-aza-bicyclo[3.2.1]oct-3-yl)-phenyl]acetonitrile (220 mg, $0.92 \mathrm{mmol}, 92 \%$ yield) as a foamy solid.

Mp.: $105-106^{\circ} \mathrm{C}$.

${ }^{\mathbf{1}} \mathbf{H}$-NMR $\left(\mathrm{CDCl}_{3}, 300 \mathrm{MHz}, 25^{\circ} \mathrm{C}\right): \delta[\mathrm{ppm}]=7.09(\mathrm{~d}, \mathrm{~J}=8.4 \mathrm{~Hz}, 2 \mathrm{H}), 6.97(\mathrm{~d}, \mathrm{~J}=8.4 \mathrm{~Hz}$, $2 \mathrm{H}), 3.48(\mathrm{~s}, 2 \mathrm{H}), 3.27-3.25(\mathrm{~m}, 2 \mathrm{H}), 2.72-2.62(\mathrm{~m}, 1 \mathrm{H}), 2.32(\mathrm{~s}, 3 \mathrm{H}), 2.14-1.98(\mathrm{~m}, 2 \mathrm{H})$, 1.67-1.64 (m, 2H), 1.50-1.45 (m, 4H).

${ }^{13} \mathrm{C}-\mathrm{NMR}\left(\mathrm{CDCl}_{3}, 75 \mathrm{MHz}, 25^{\circ} \mathrm{C}\right): \delta[\mathrm{ppm}]=144.2,131.1,128.3,128.2,118.3,63.5,39.8$, $37.8,33.7,25.5,23.3$.

MS (EI, $70 \mathrm{eV}), \mathrm{m} / \mathrm{z}(\%): 241(100)\left[\mathrm{M}+\mathrm{H}^{+}\right], 235$ (1), 102 (26), 51 (1).

HRMS: calculated for $\mathrm{C}_{16} \mathrm{H}_{21} \mathrm{~N}_{2}{ }^{+} 241.1699$, found 241.1691 .

IR (ATR): $\ddot{v}\left[\mathrm{~cm}^{-1}\right]=2934$ (s), 2910 (s), 2798 (m), 2244 (w), 1512 (s), 1418 (s), 1354 (s), 1122 (s), 1052 (s), 1036 (s), 848 (vs), 800 (s), 786 (s), 768 (vs), 706 (m), 630 (m).

exo-2-(8-Methyl-8-aza-bicyclo[3.2.1]oct-3-yl)quinoline (3q)

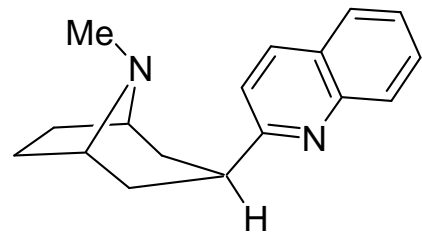

Trifluoromethanesulfonic acid quinolin-2-yl ester $(277 \mathrm{mg}, 1.0 \mathrm{mmol})$ was reacted with 8methyl-8-aza-bicyclo[3.2.1] octyl-3-magnesium chloride (0.3 M in THF, $4.0 \mathrm{~mL}, 1.2 \mathrm{mmol})$ at $25^{\circ} \mathrm{C}$ for $72 \mathrm{~h}$, according to TP2. Aqueous workup and purification by flash chromatography $\left(\mathrm{CH}_{2} \mathrm{Cl}_{2}\right.$ /methanol, 20:1) furnished exo-2-(8-methyl-8-aza-bicyclo[3.2.1]oct-3-yl)-quinoline (202 $\mathrm{mg}, 0.80 \mathrm{mmol}, 80 \%$ yield) as an orange-brown oil.

${ }^{1} \mathbf{H}-\mathbf{N M R}\left(\mathrm{CDCl}_{3}, 300 \mathrm{MHz}, 25^{\circ} \mathrm{C}\right): \delta[\mathrm{ppm}]=7.95(\mathrm{t}, \mathrm{J}=9.0 \mathrm{~Hz}, 2 \mathrm{H}), 7.64(\mathrm{~d}, \mathrm{~J}=7.9 \mathrm{~Hz}$, $1 \mathrm{H}), 7.58-7.53(\mathrm{~m}, 1 \mathrm{H}), 7.38-7.33(\mathrm{~m}, 2 \mathrm{H}), 3.22-3.11(\mathrm{~m}, 3 \mathrm{H}), 2.28(\mathrm{~s}, 3 \mathrm{H}), 2.11-1.99$ (m, $4 \mathrm{H}), 1.73-1.68(\mathrm{~m}, 4 \mathrm{H})$.

${ }^{13} \mathrm{C}-\mathrm{NMR}\left(\mathrm{CDCl}_{3}, 75 \mathrm{MHz}, 25^{\circ} \mathrm{C}\right): \delta[\mathrm{ppm}]=165.4,147.9,136.7,129 ., 129.1,127.6,127.2$, $126.0,119.8,61.8,40.6,38.5,37.6,36.2$.

MS (EI, $70 \mathrm{eV}), \mathrm{m} / \mathrm{z}(\%): 252$ (87) [M+], 167 (61), 157 (100), 143 (42), 130 (20), 96 (31), 83 (62).

HRMS: calculated for $\mathrm{C}_{17} \mathrm{H}_{20} \mathrm{~N}_{2}$ 252.1626, found 252.1610.

IR (ATR): $\ddot{v}\left[\mathrm{~cm}^{-1}\right]=2958(\mathrm{vs}), 2796(\mathrm{~m}), 2672(\mathrm{~m}), 2572(\mathrm{~s}), 2548$ (s), $2494(\mathrm{~m}), 1600$ (s), 1562 (m), 1502 (s), 1476 (s), 1454 (s), 1426 (s), 1396 (m), 1032 (s), 842 (m), 816 (s), 766 (s). 


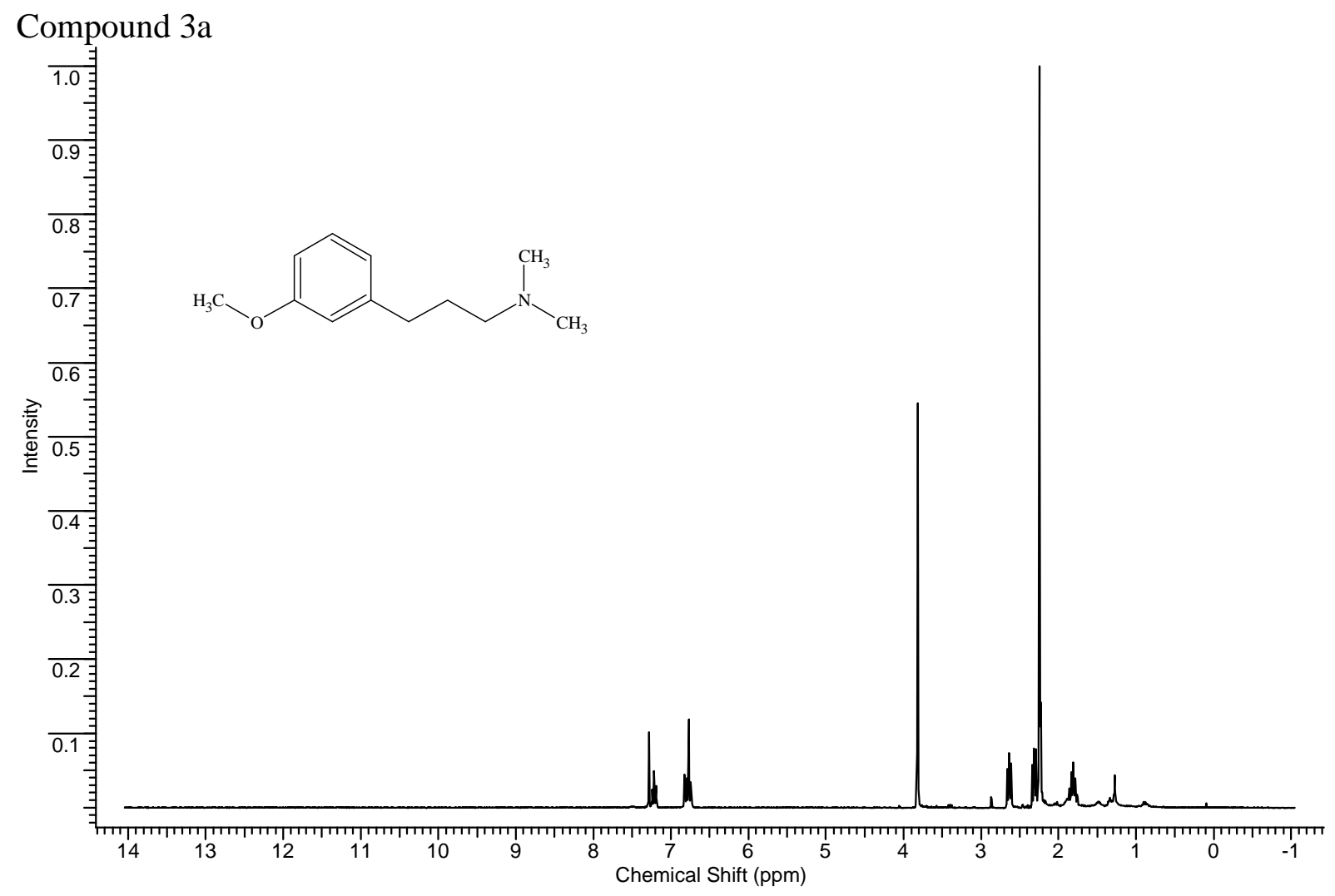

Compound 3a

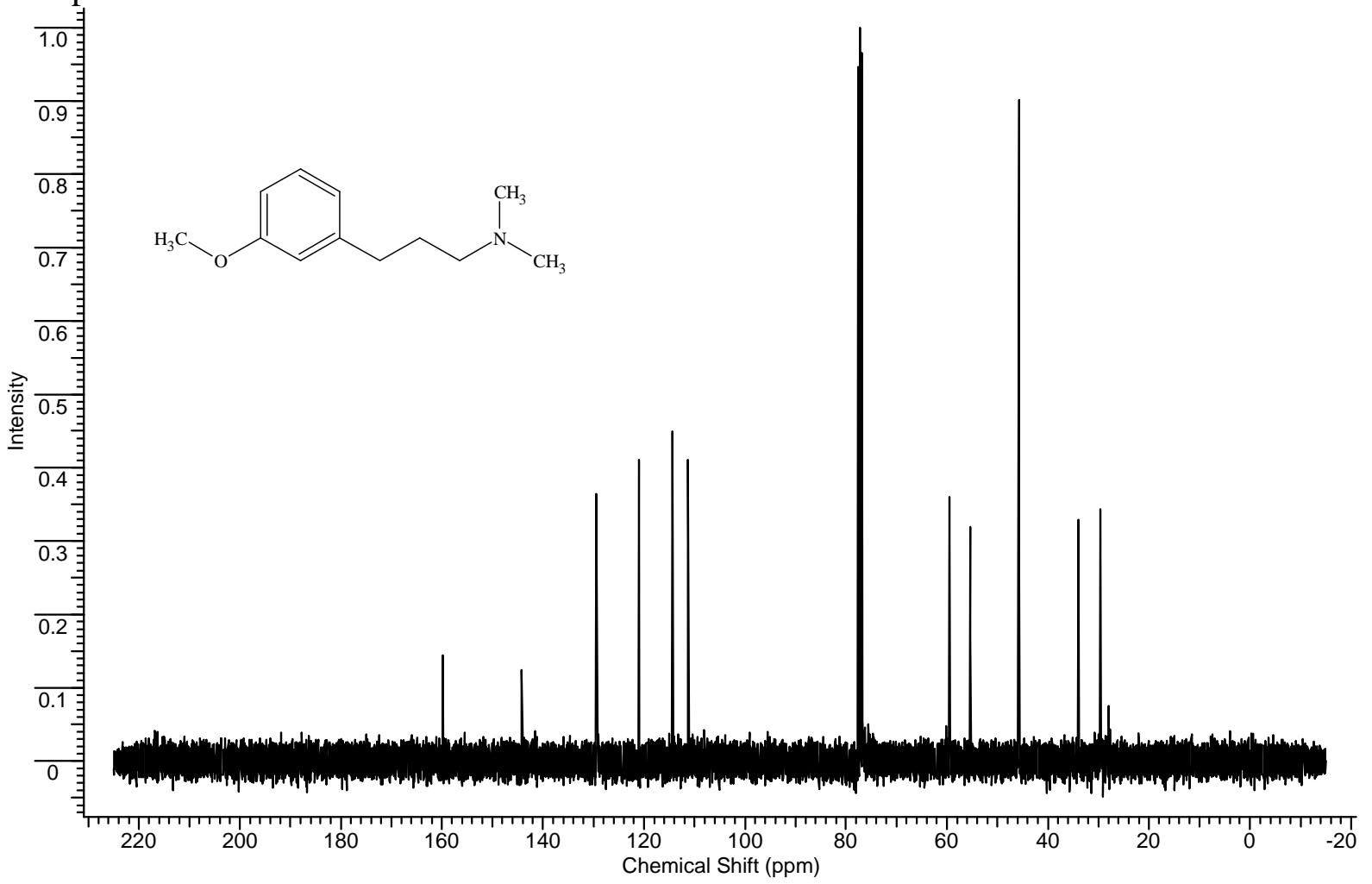




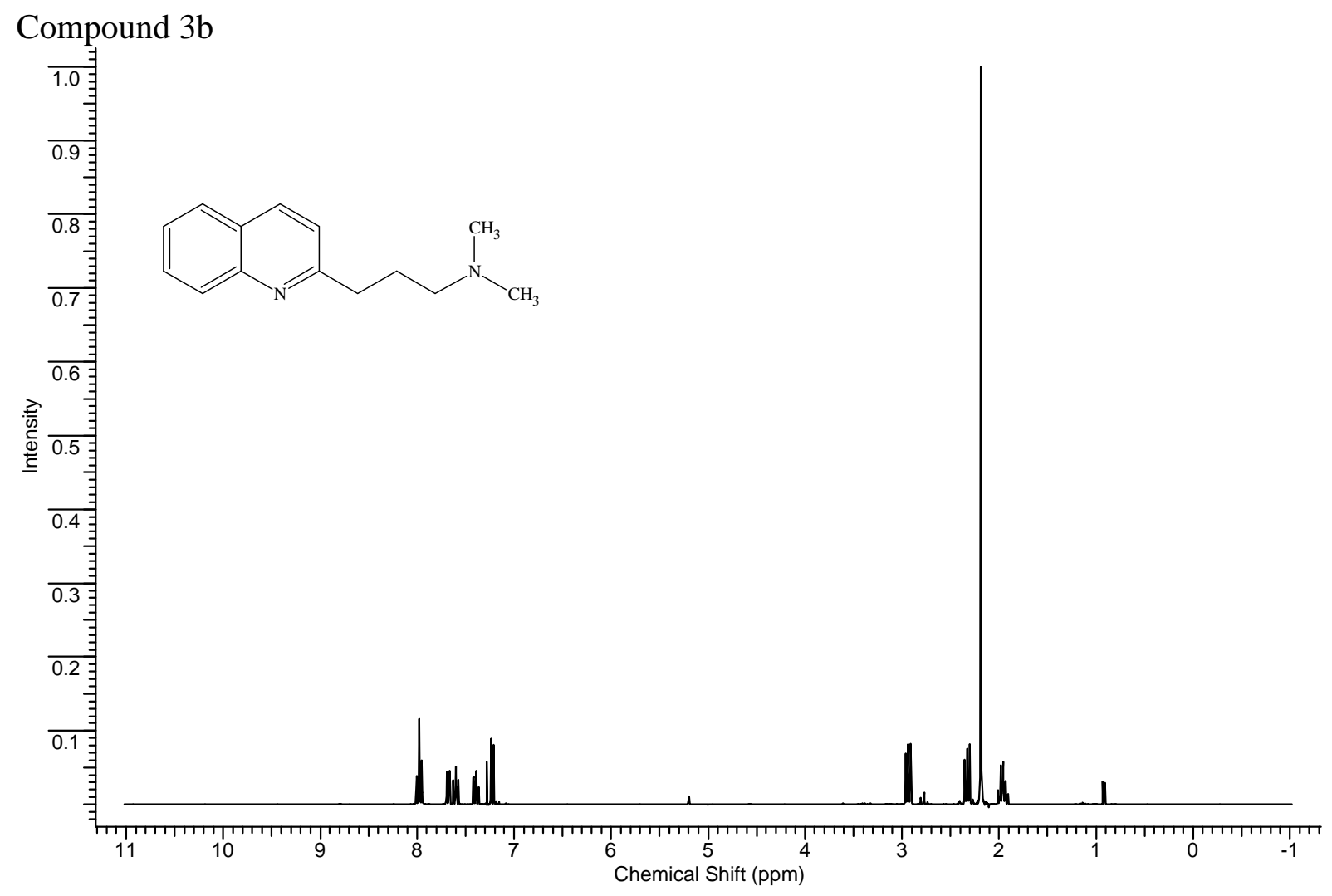

\section{Compound $3 b$}

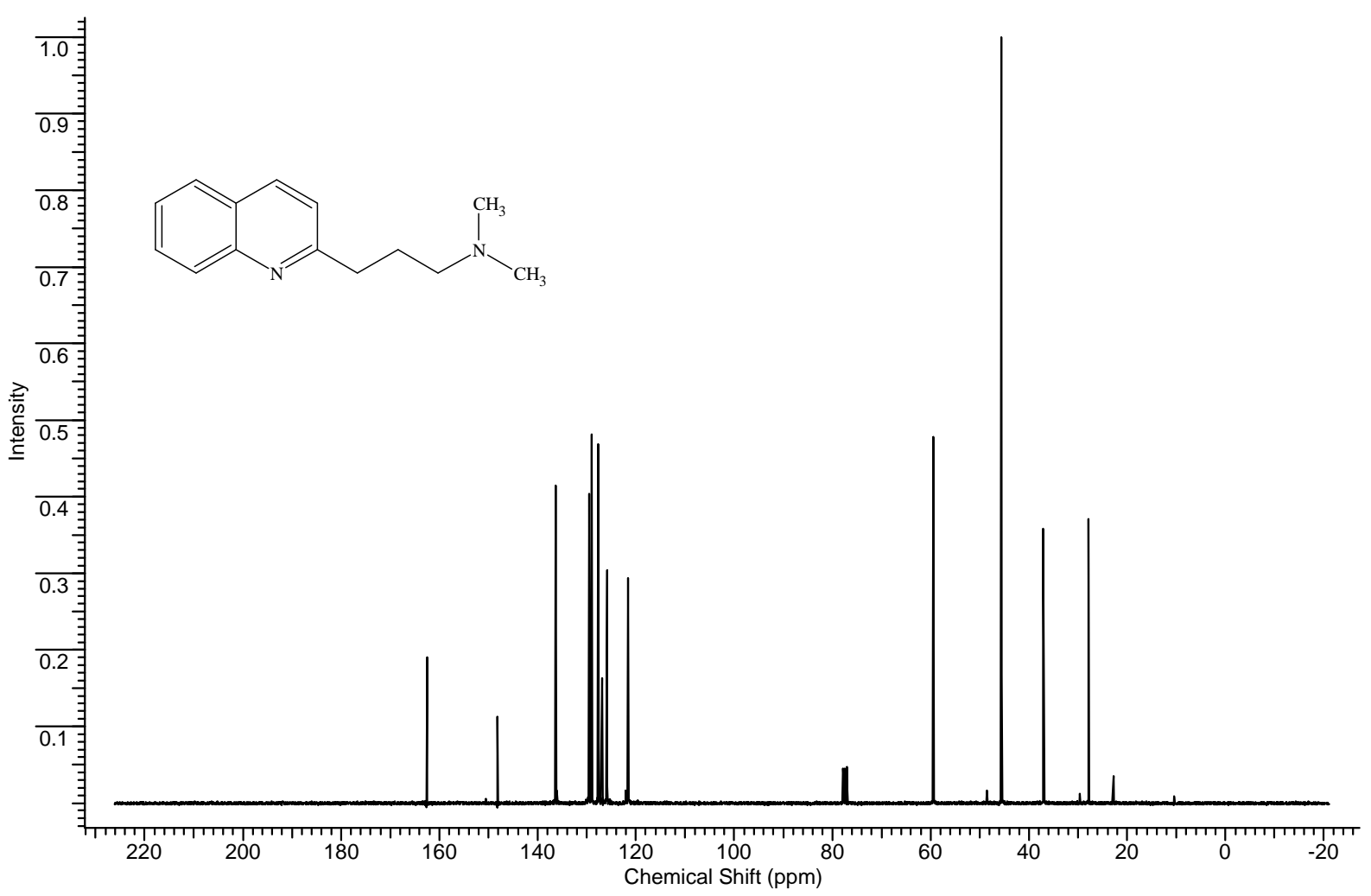



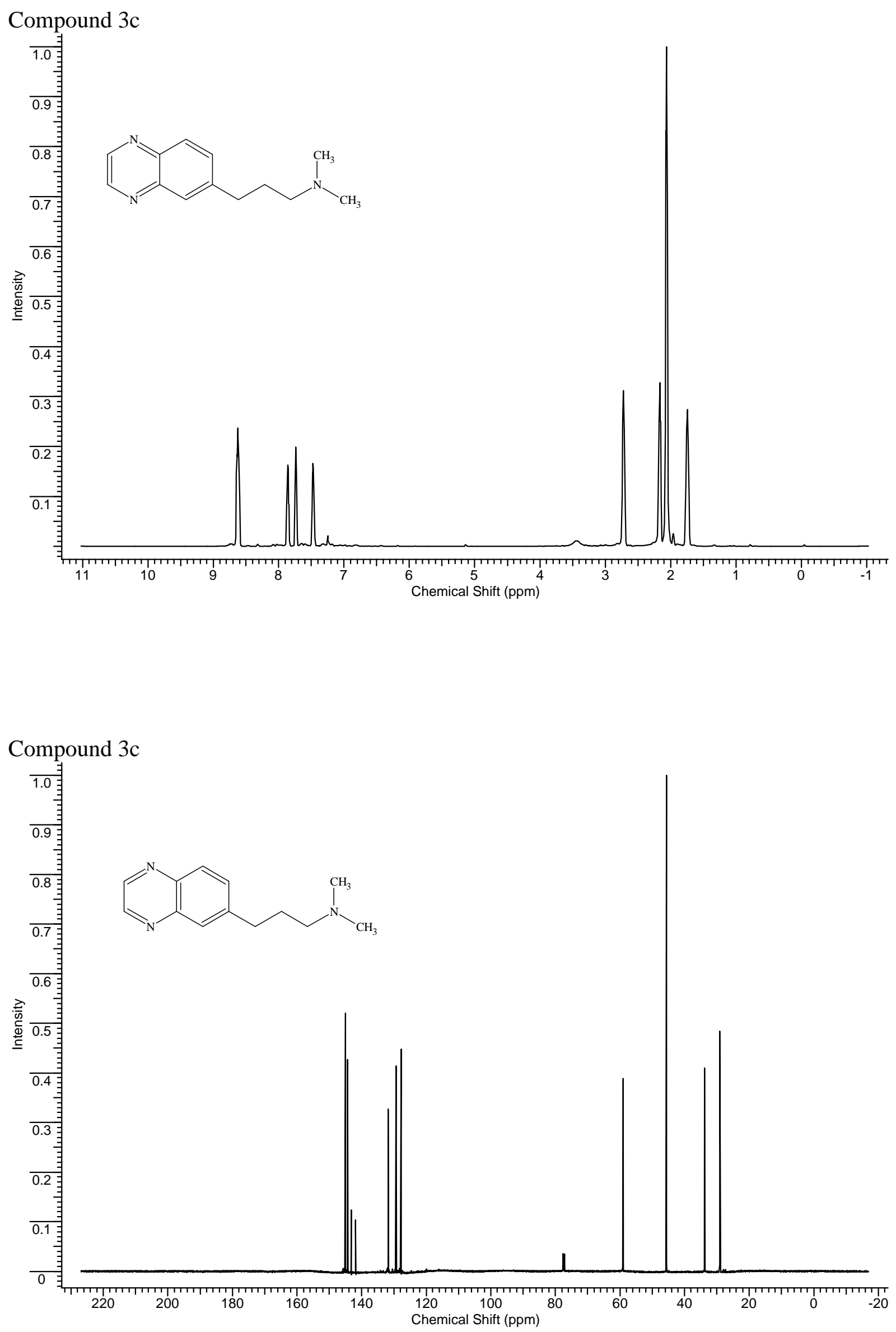

S16 


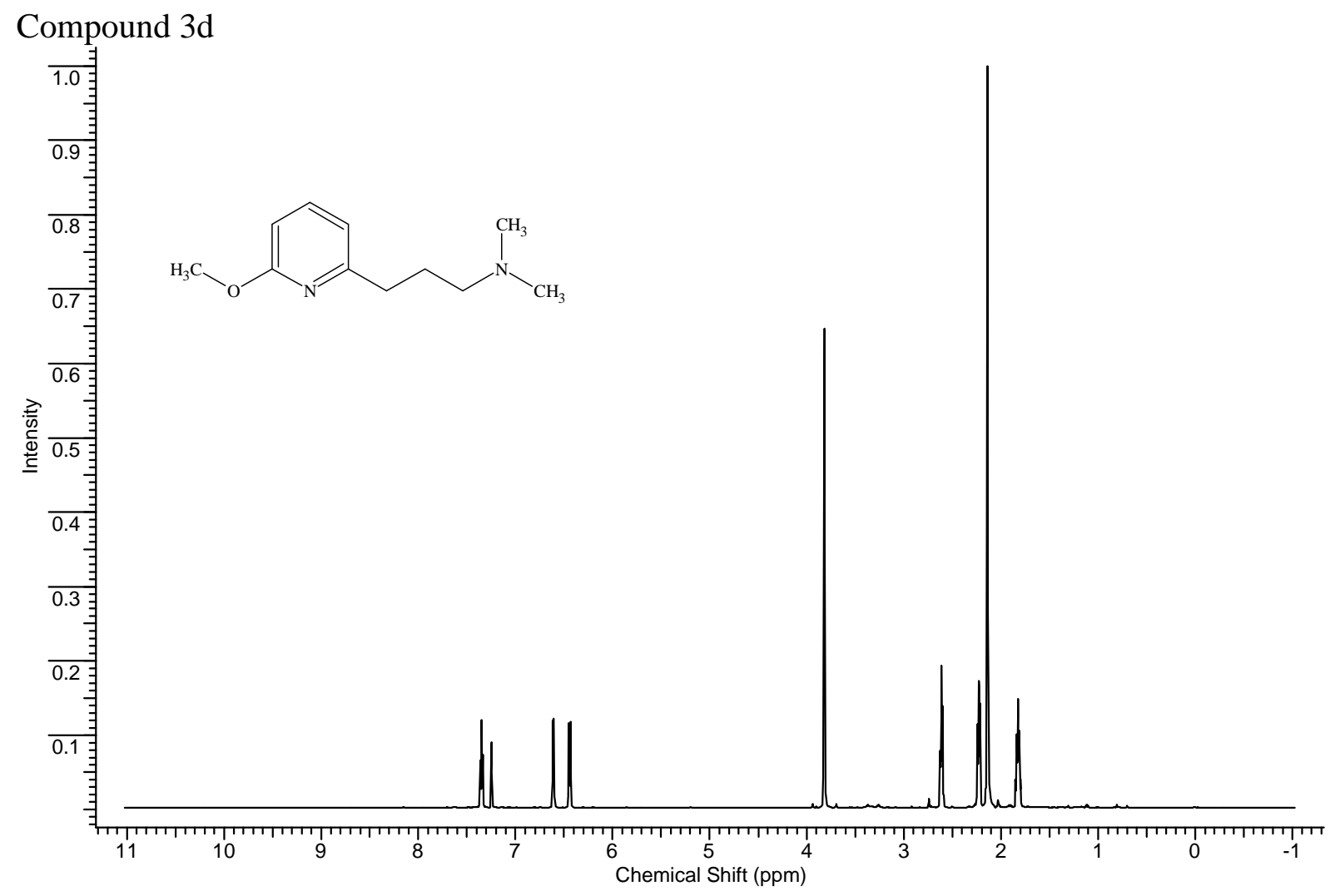

\section{Compound 3d}

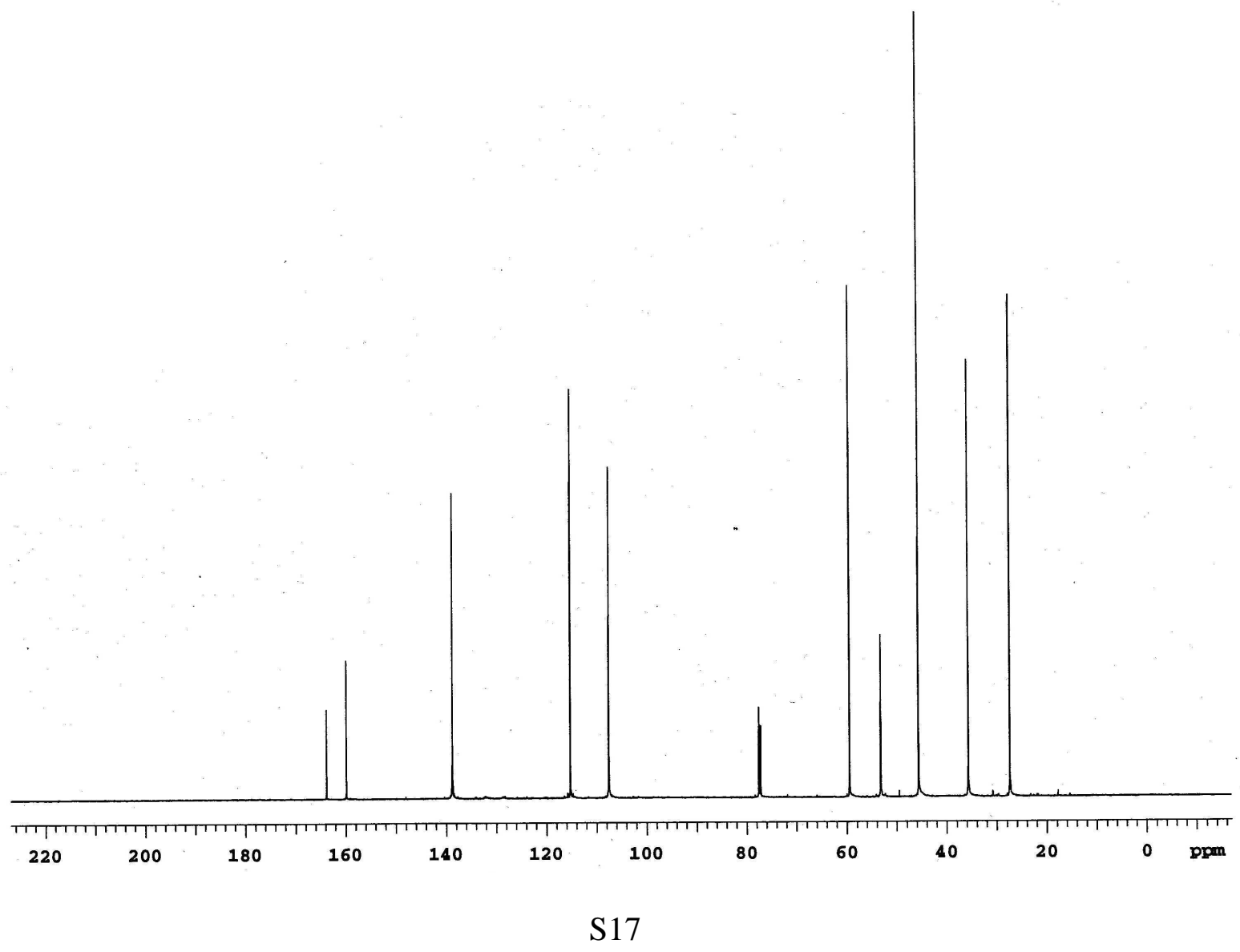



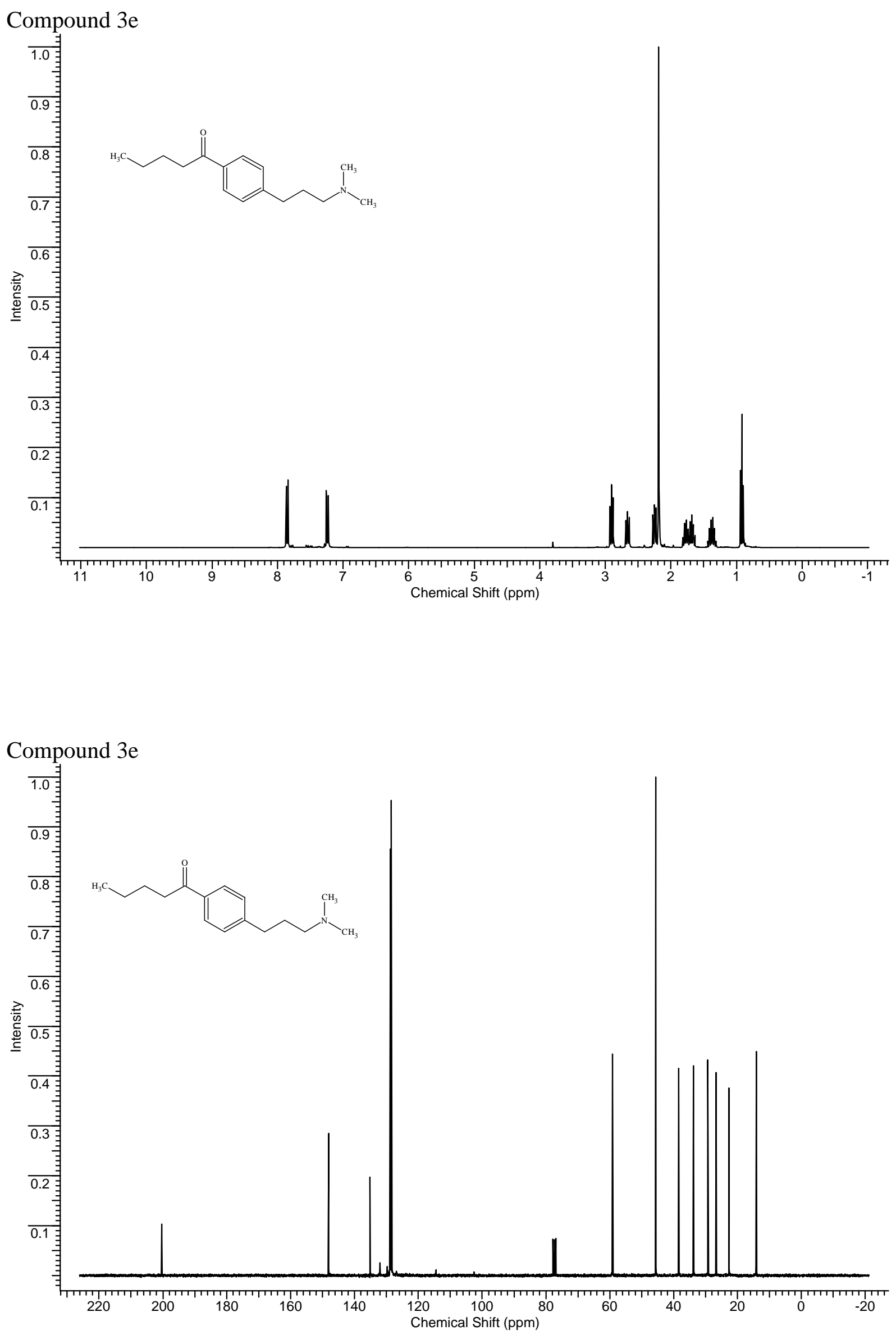

S18 


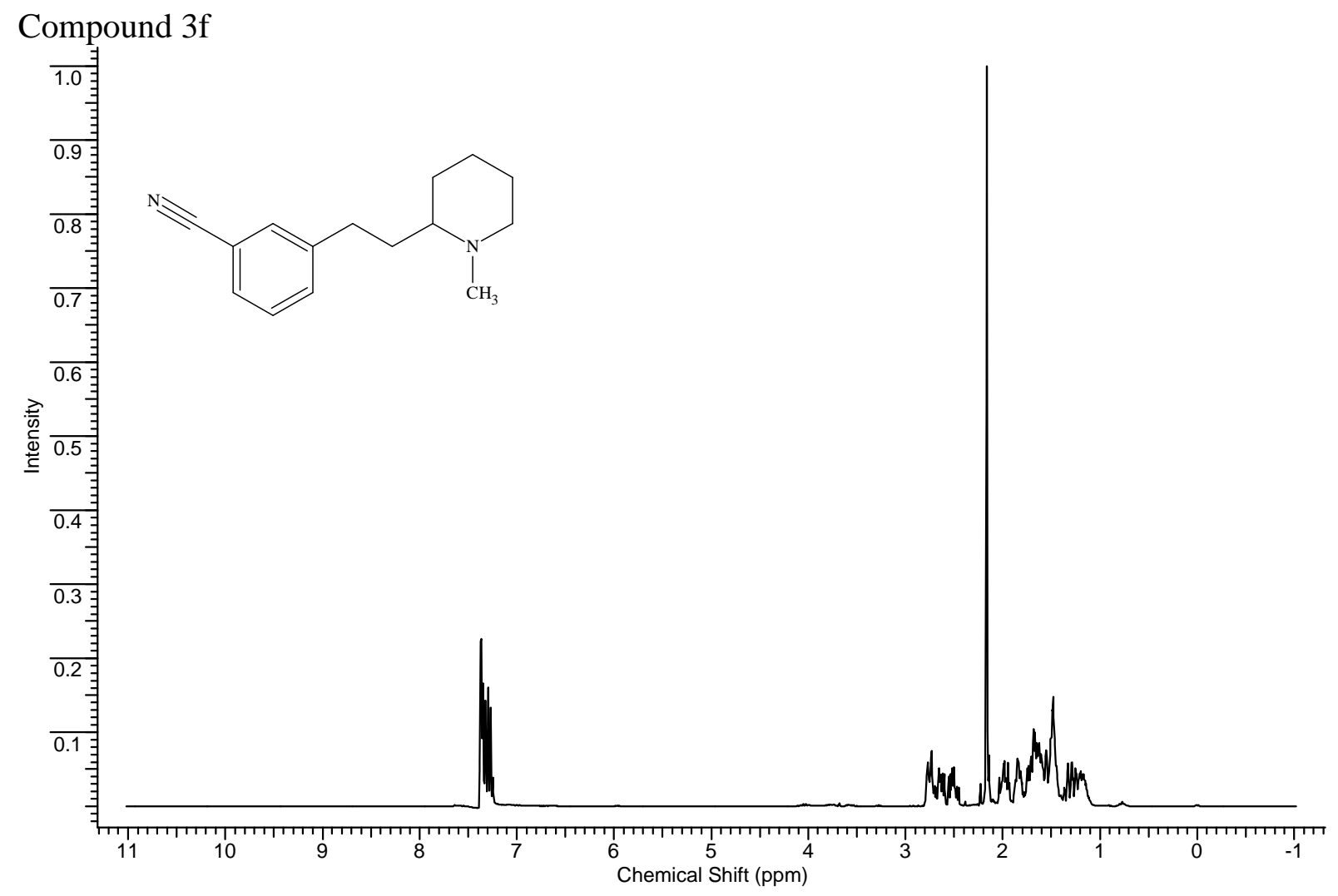

\section{Compound $3 \mathrm{f}$}

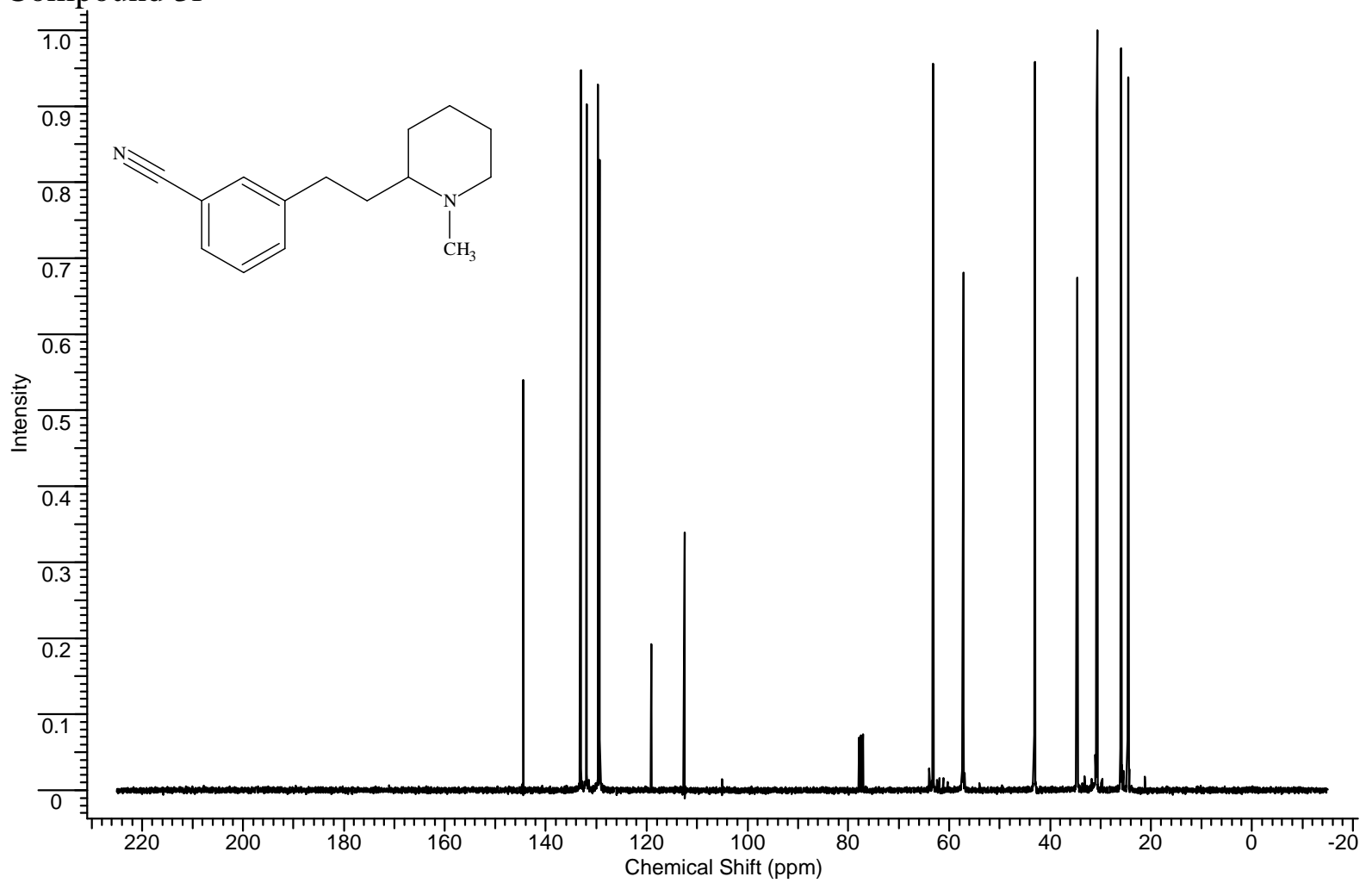




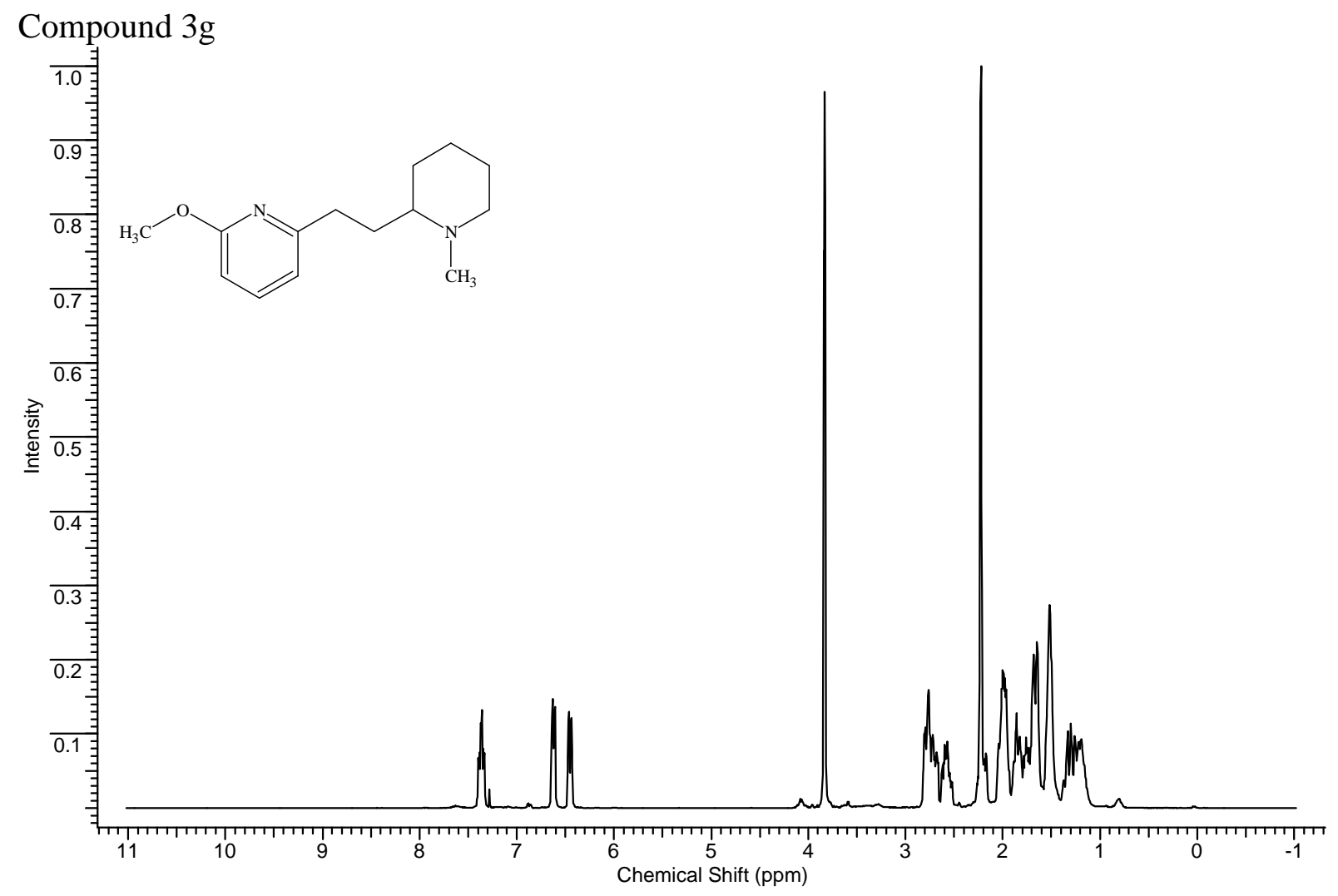

Compound 3g

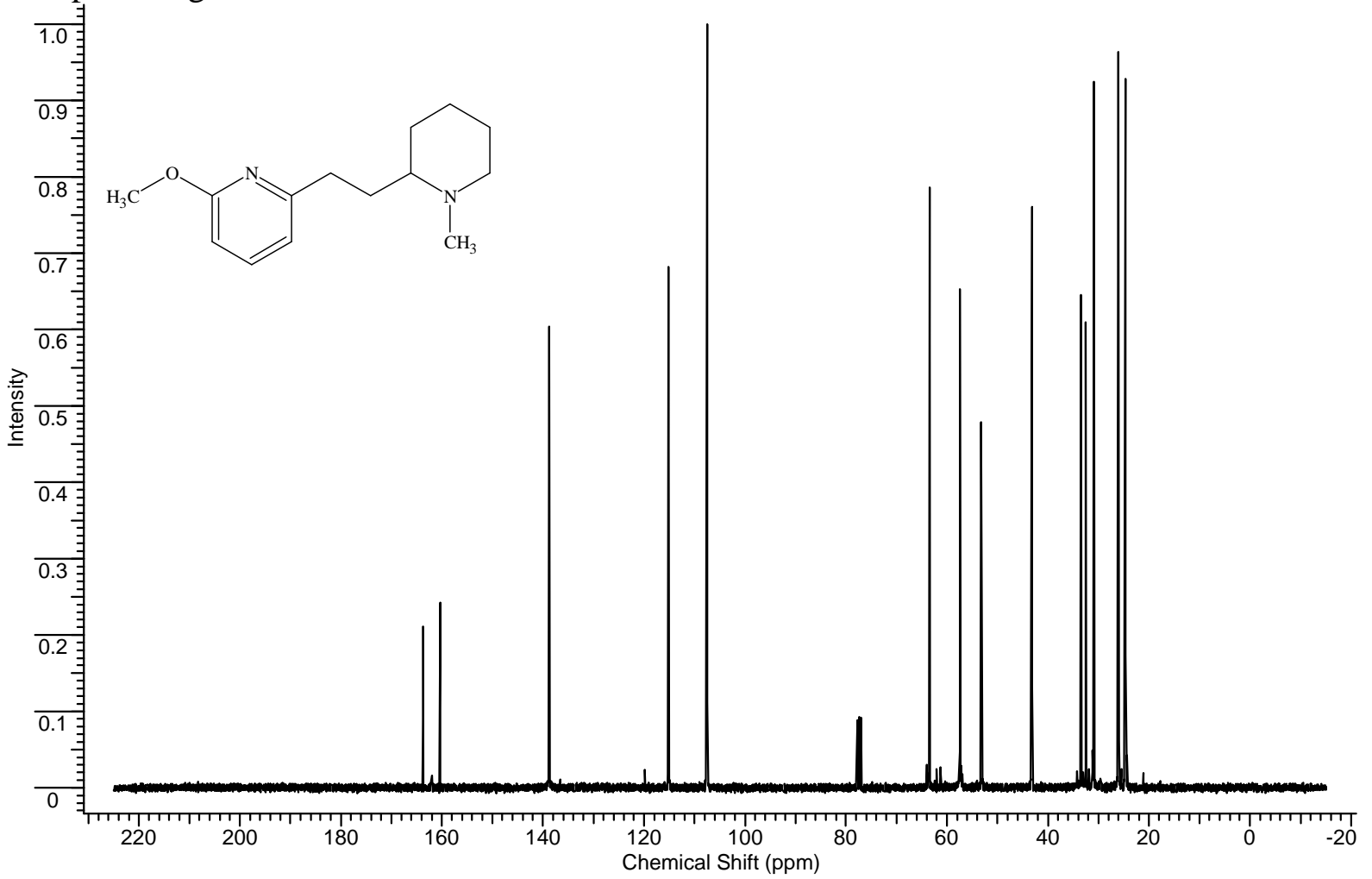




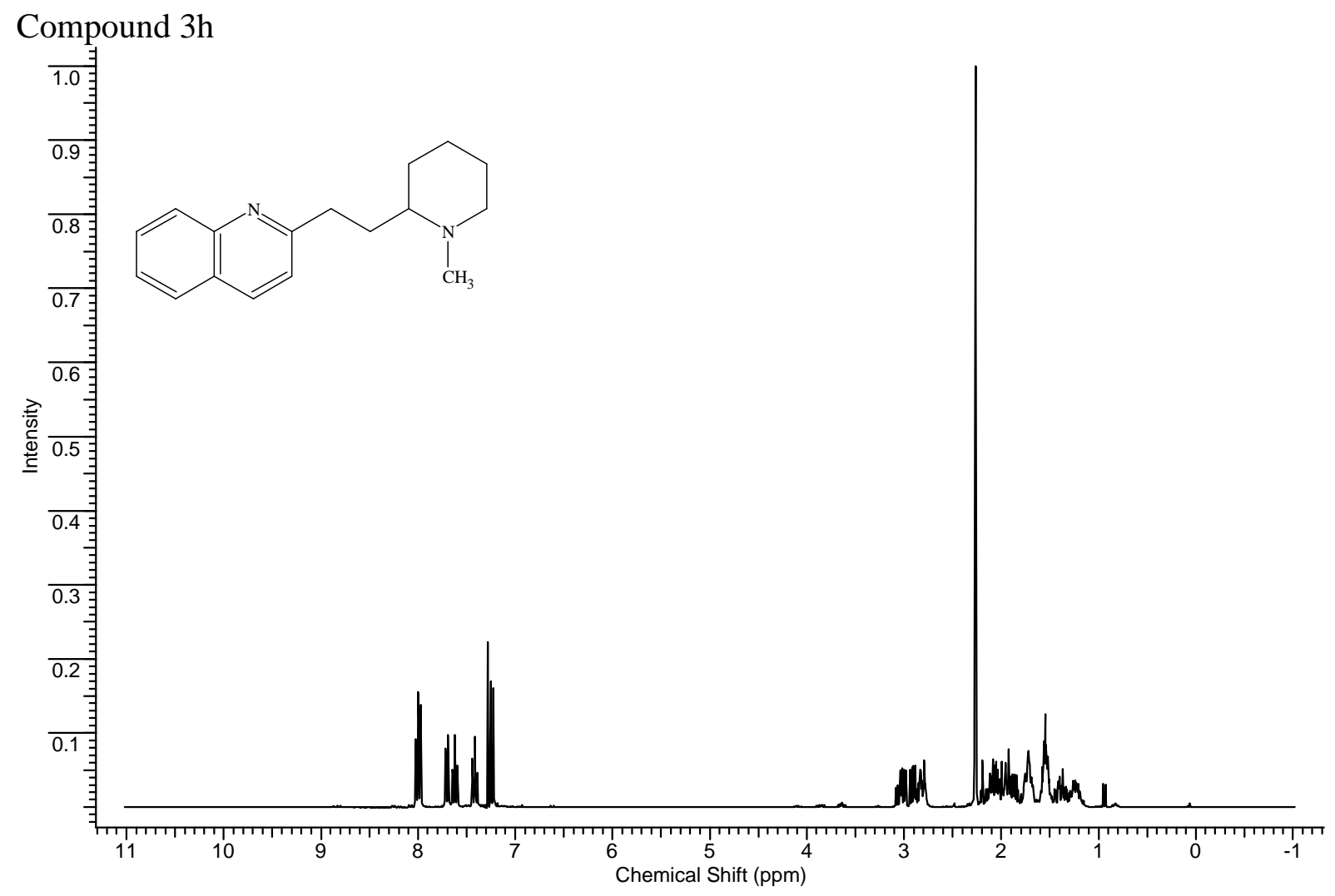

Compound $3 \mathrm{~h}$

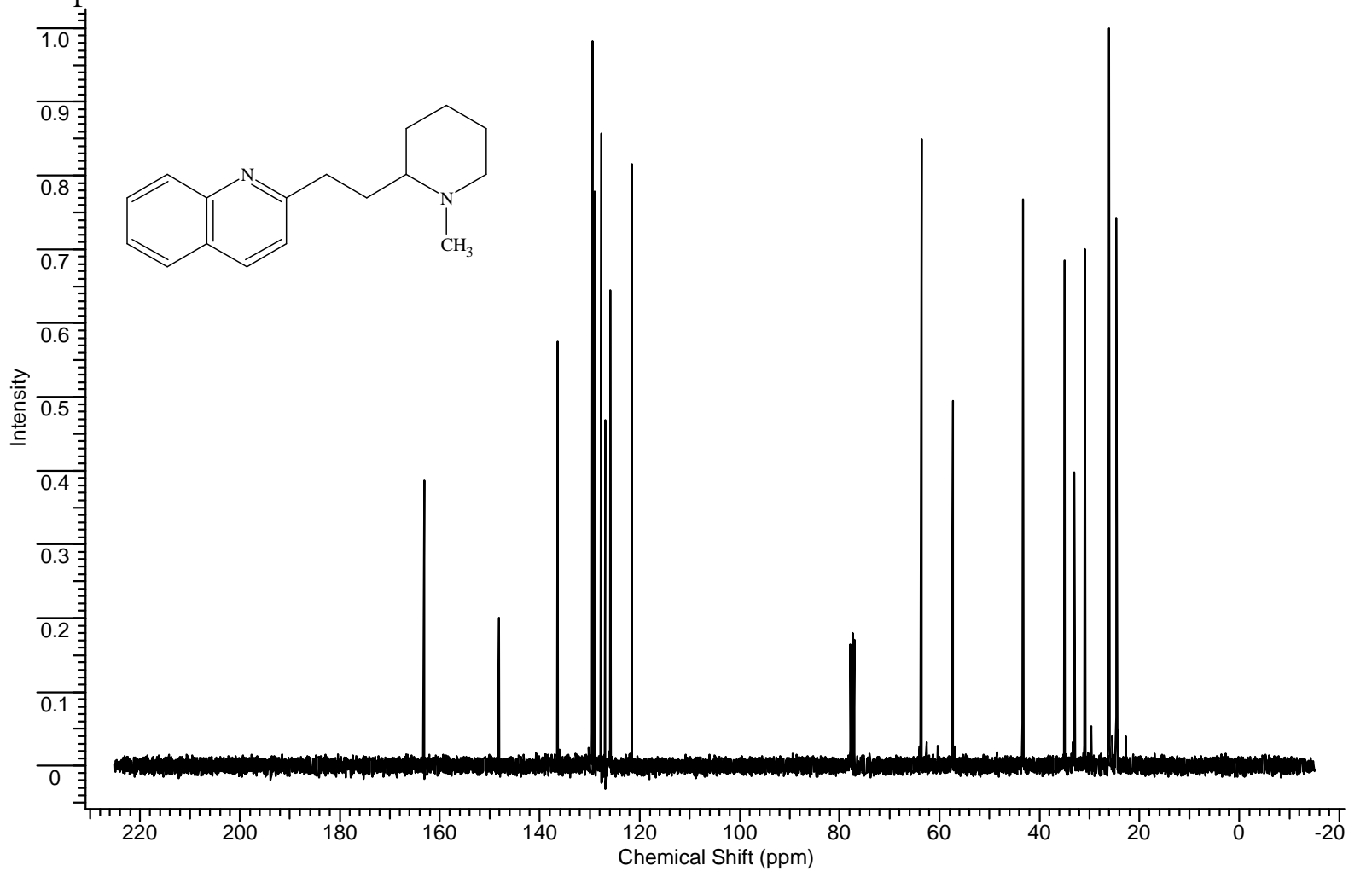




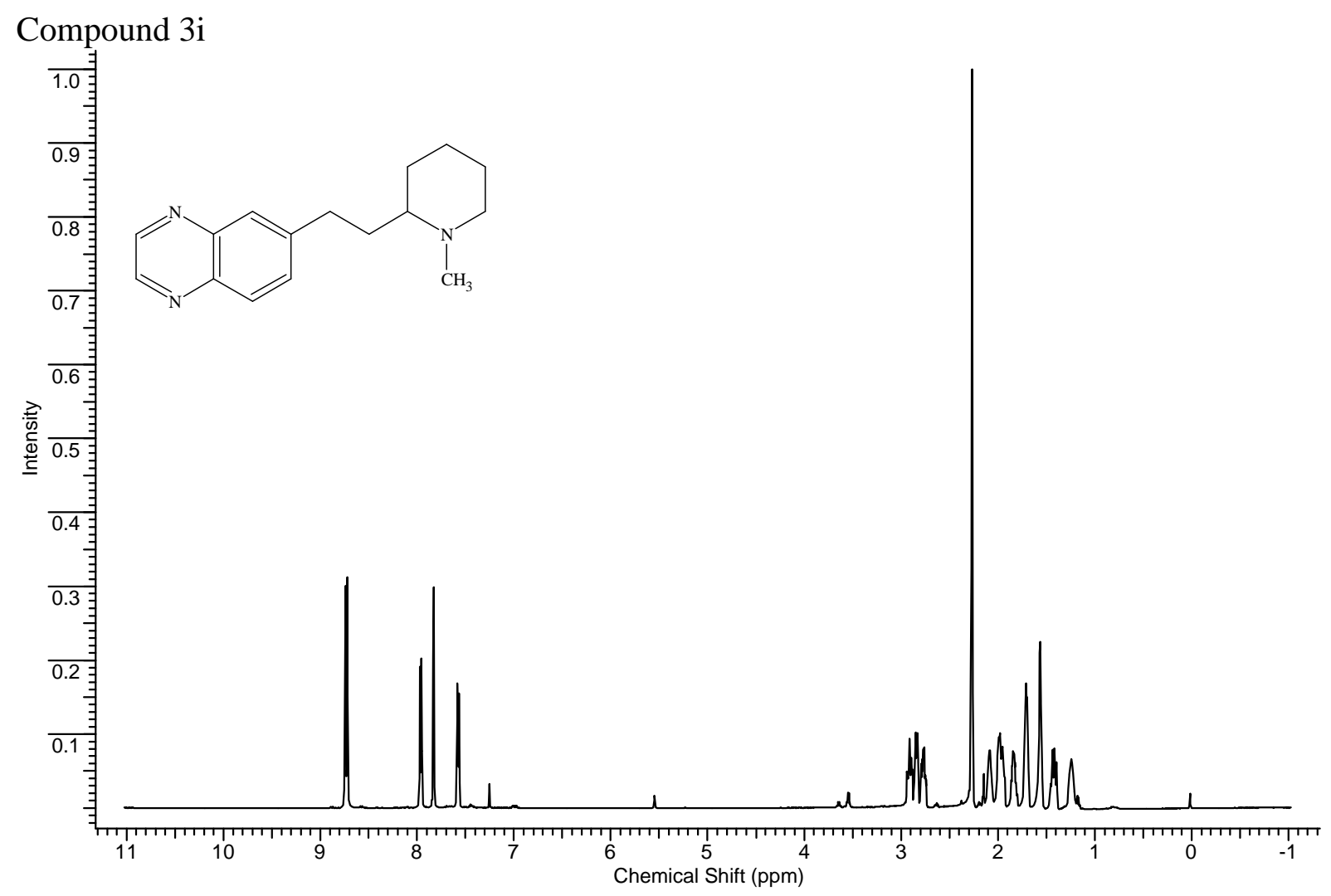

Compound $3 \mathrm{i}$

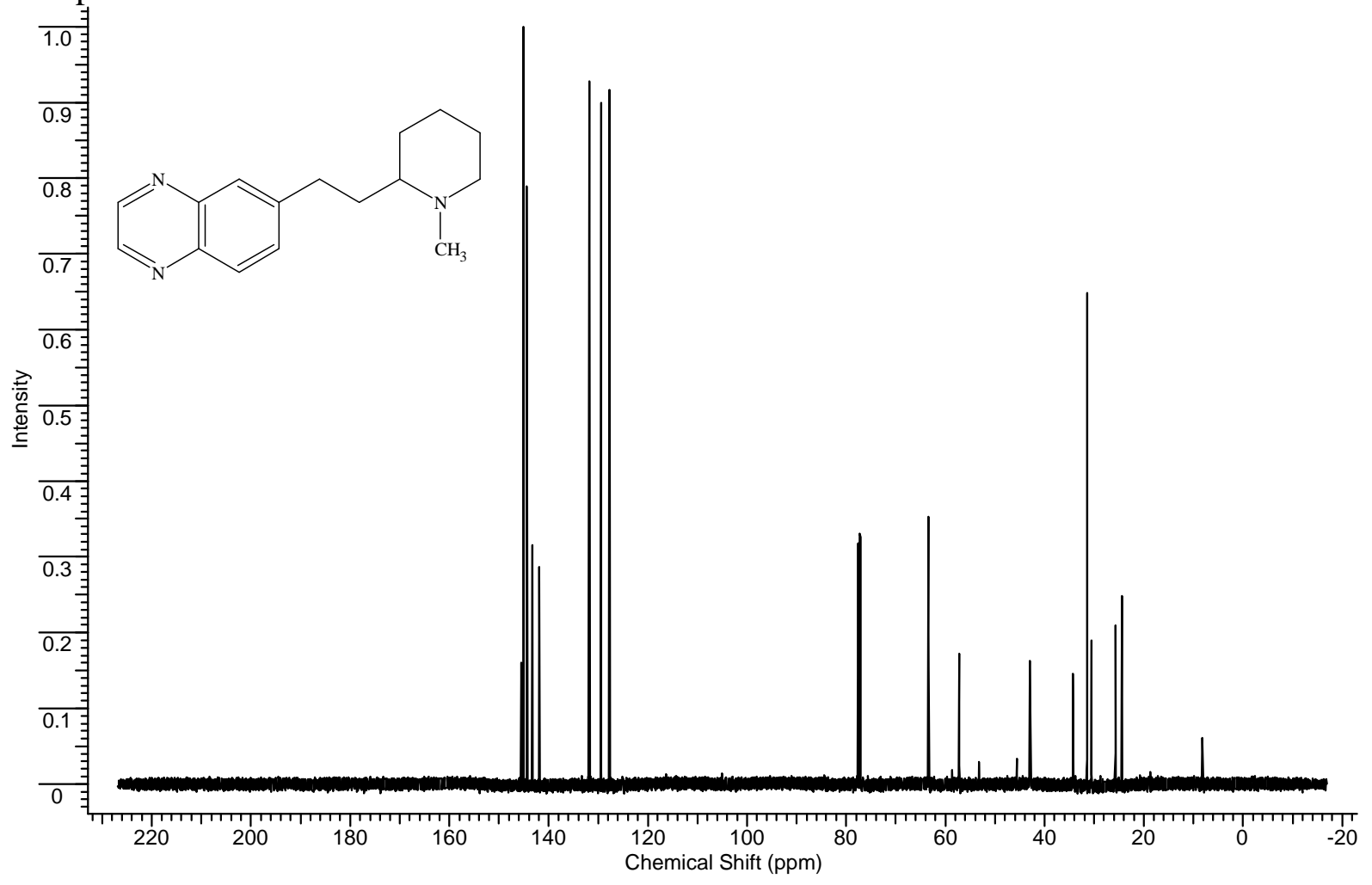




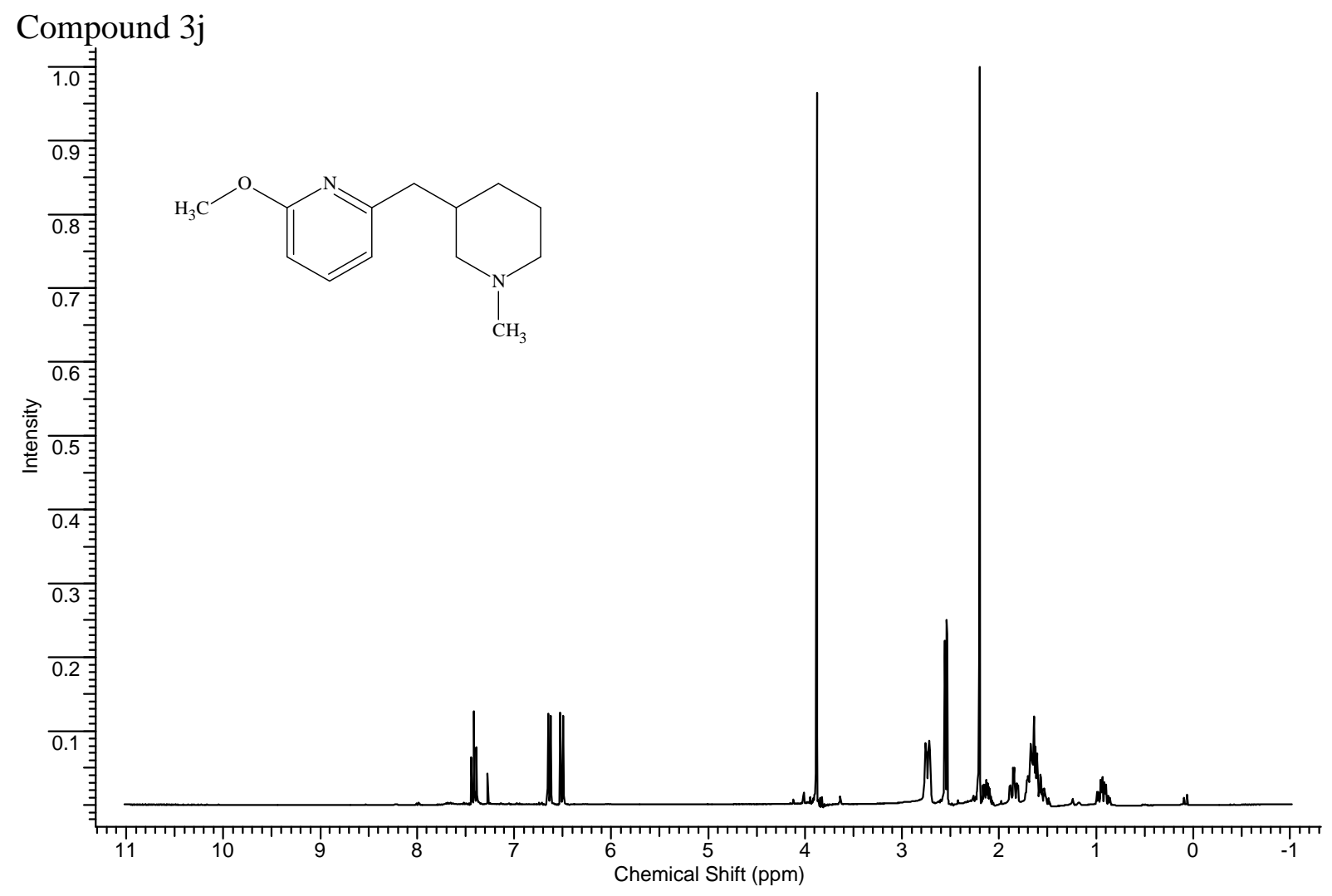

Compound $3 \mathrm{j}$

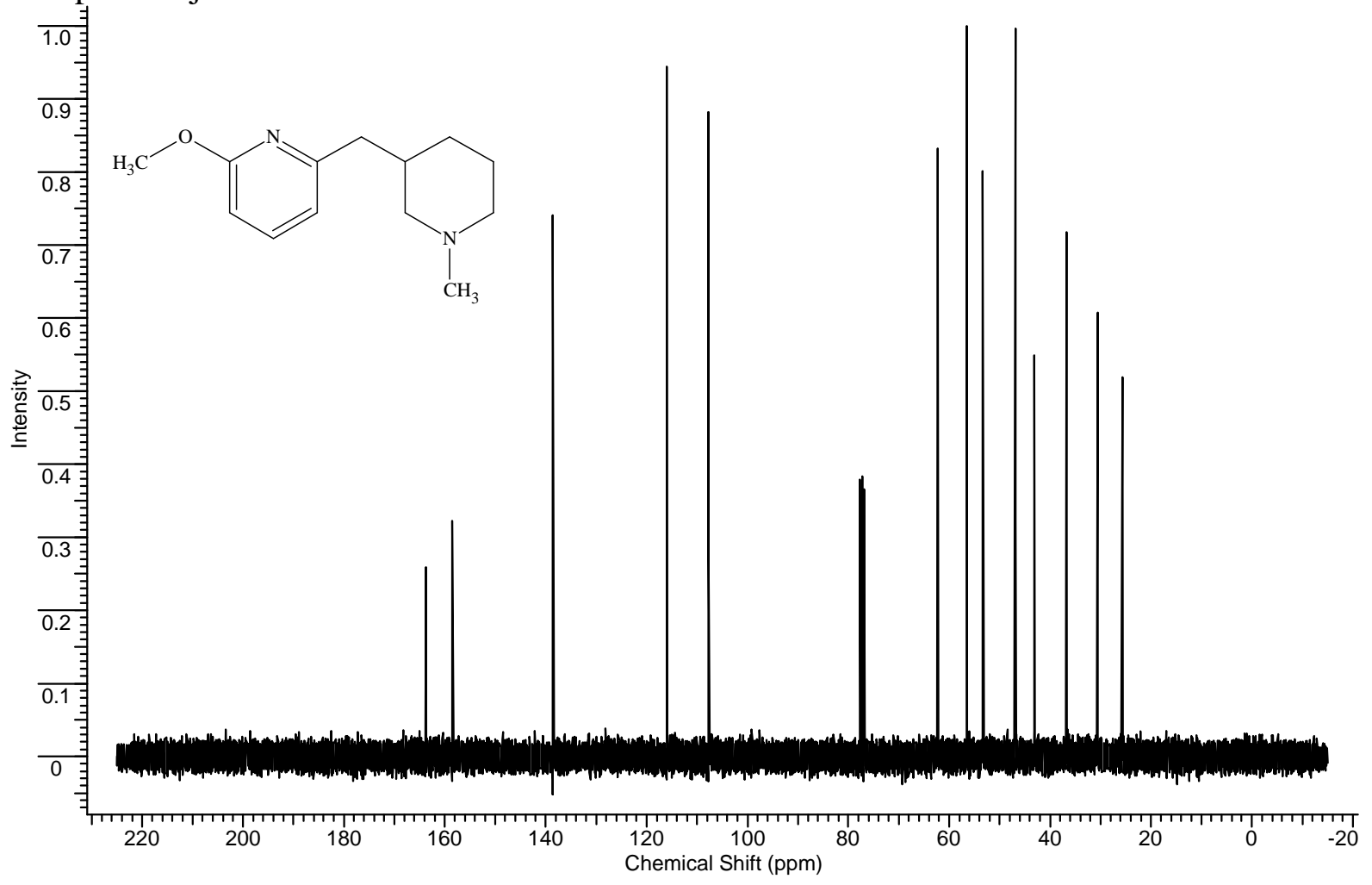



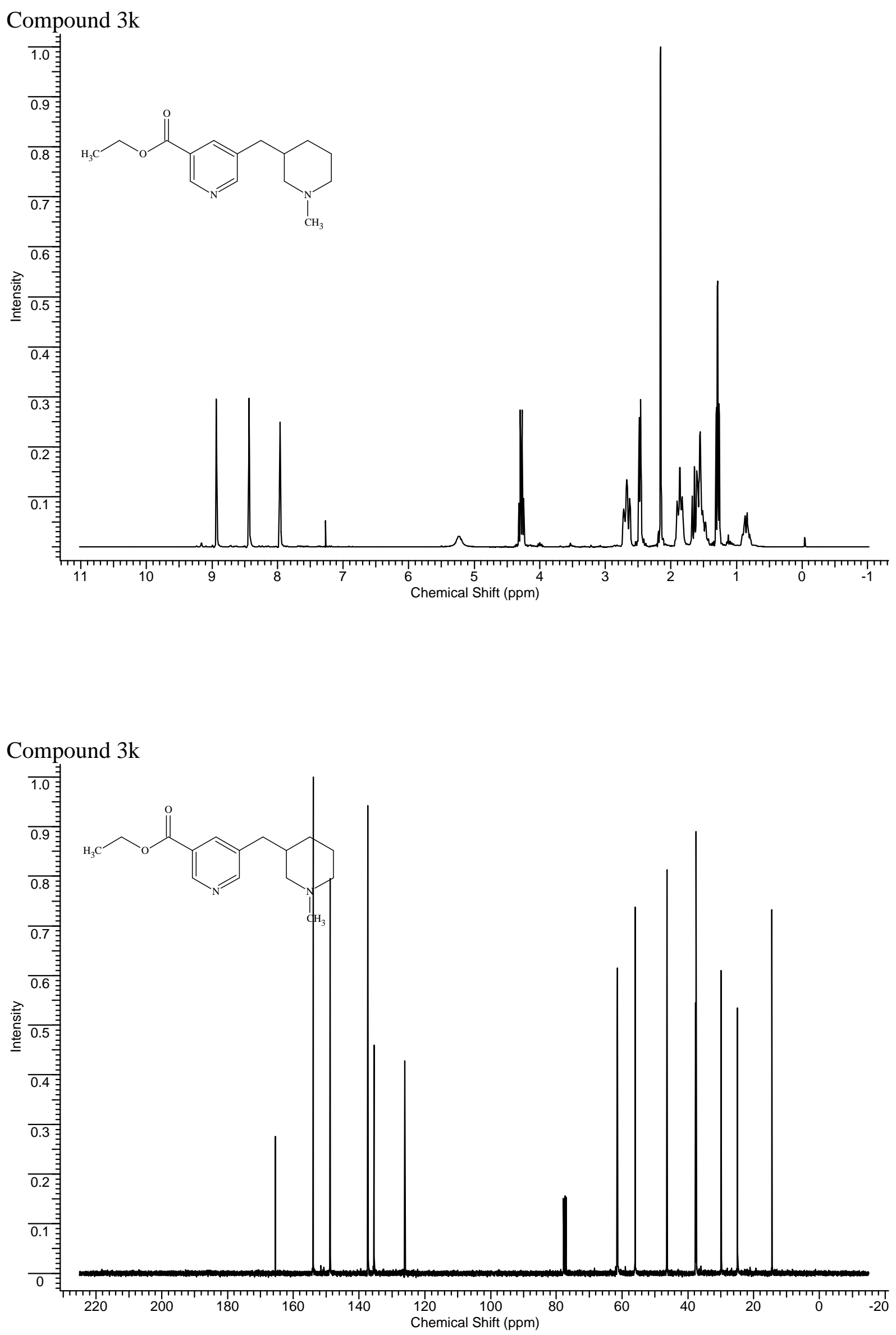

S24 


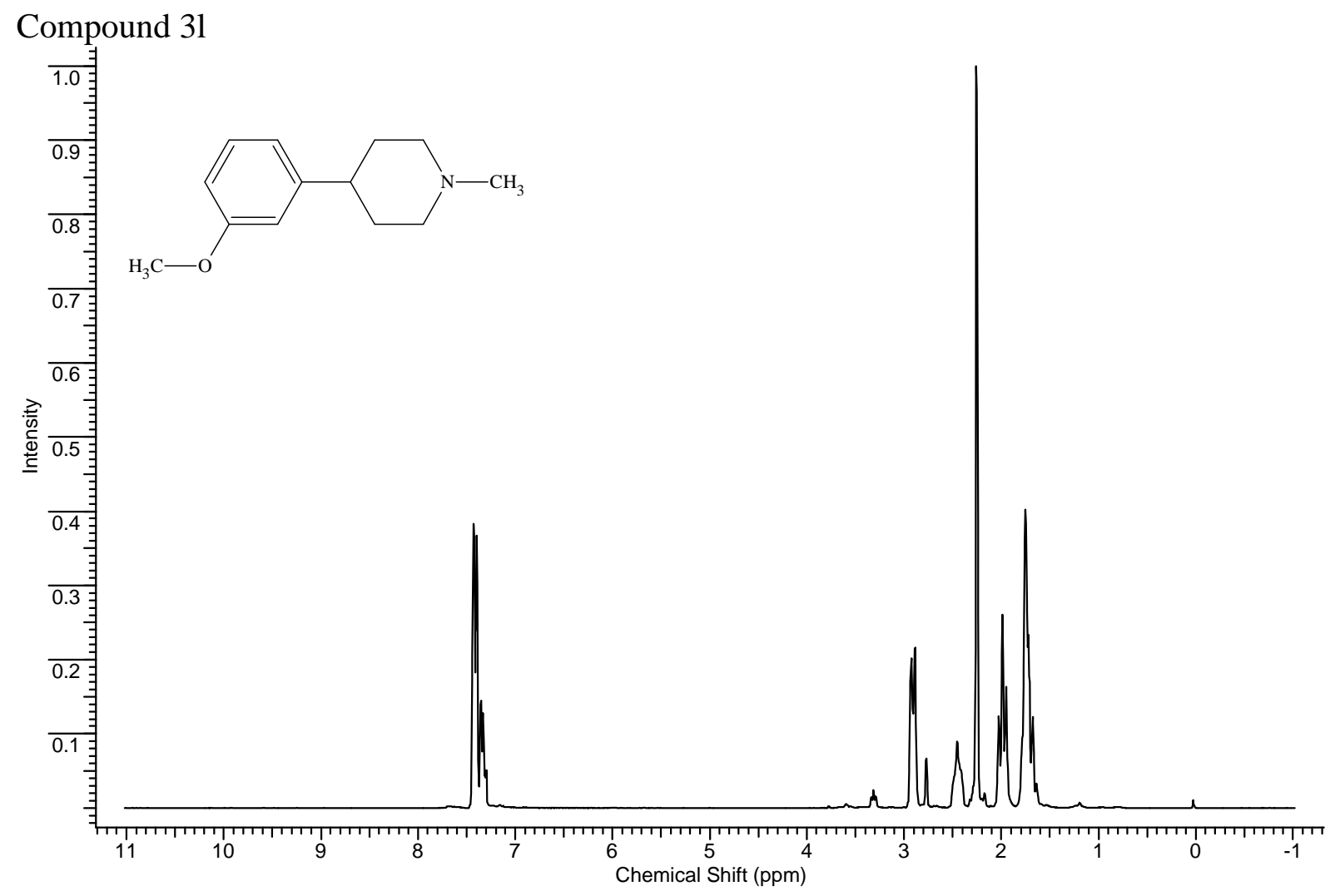

Compound 31

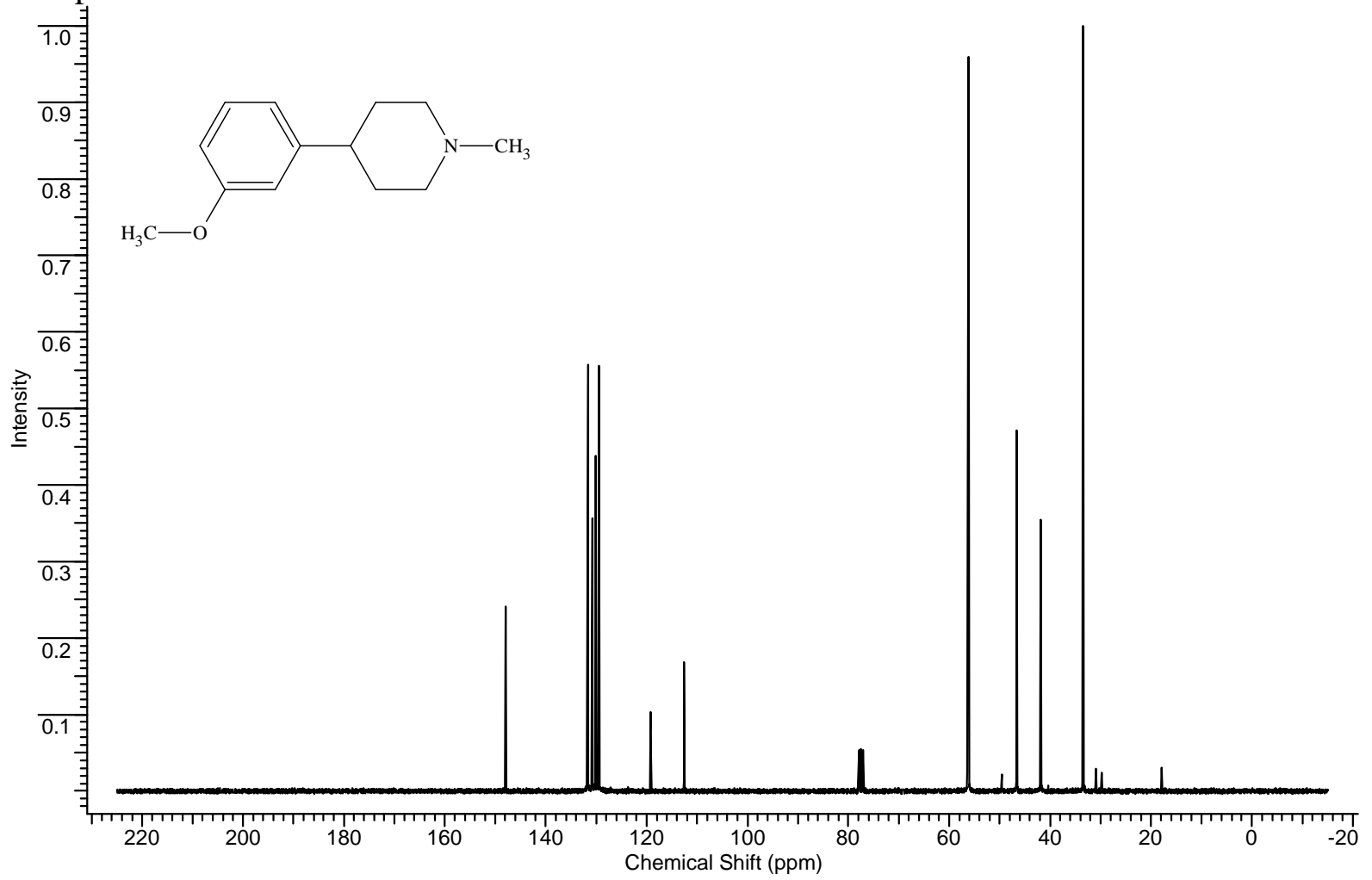




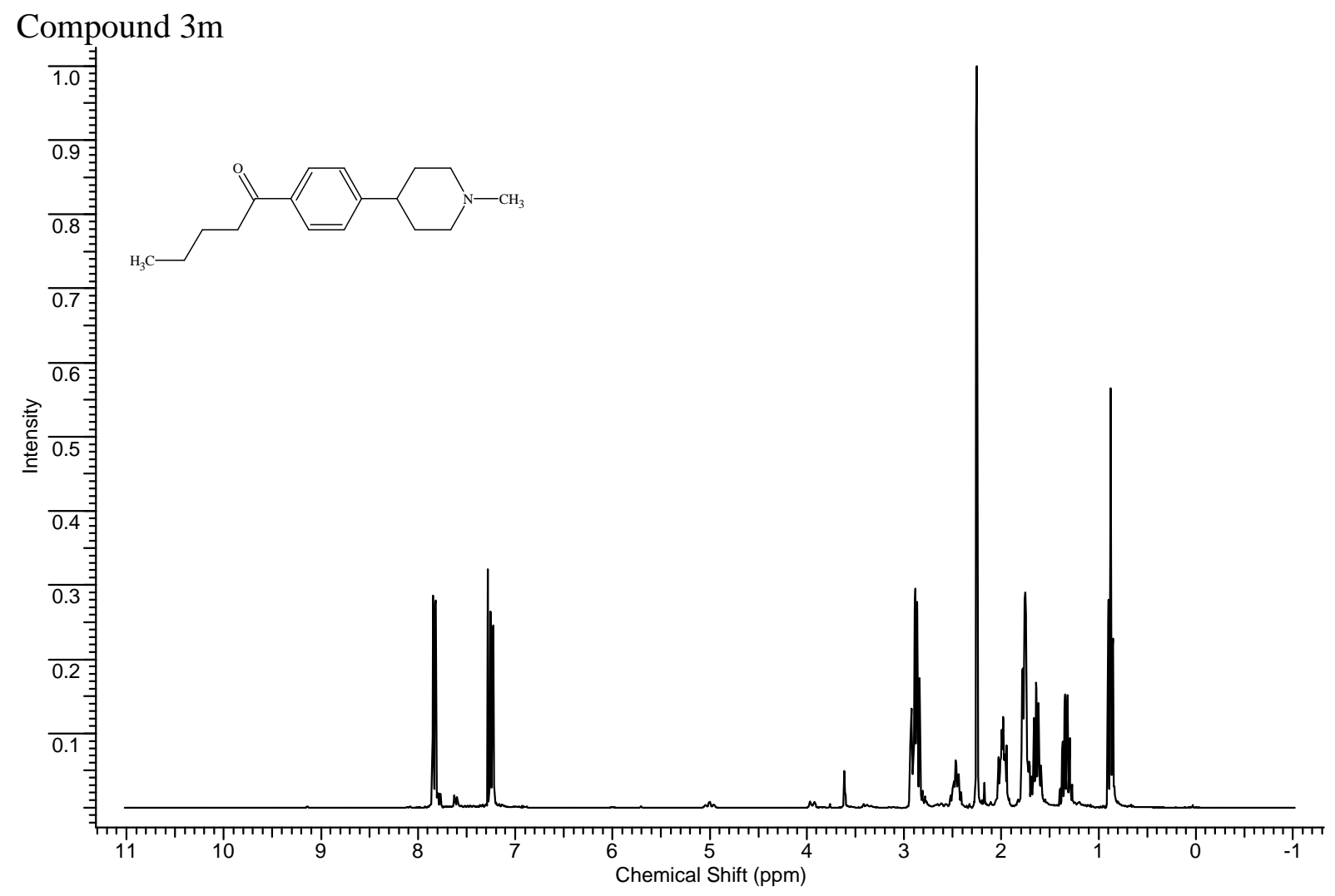

\section{Compound $3 \mathrm{~m}$}

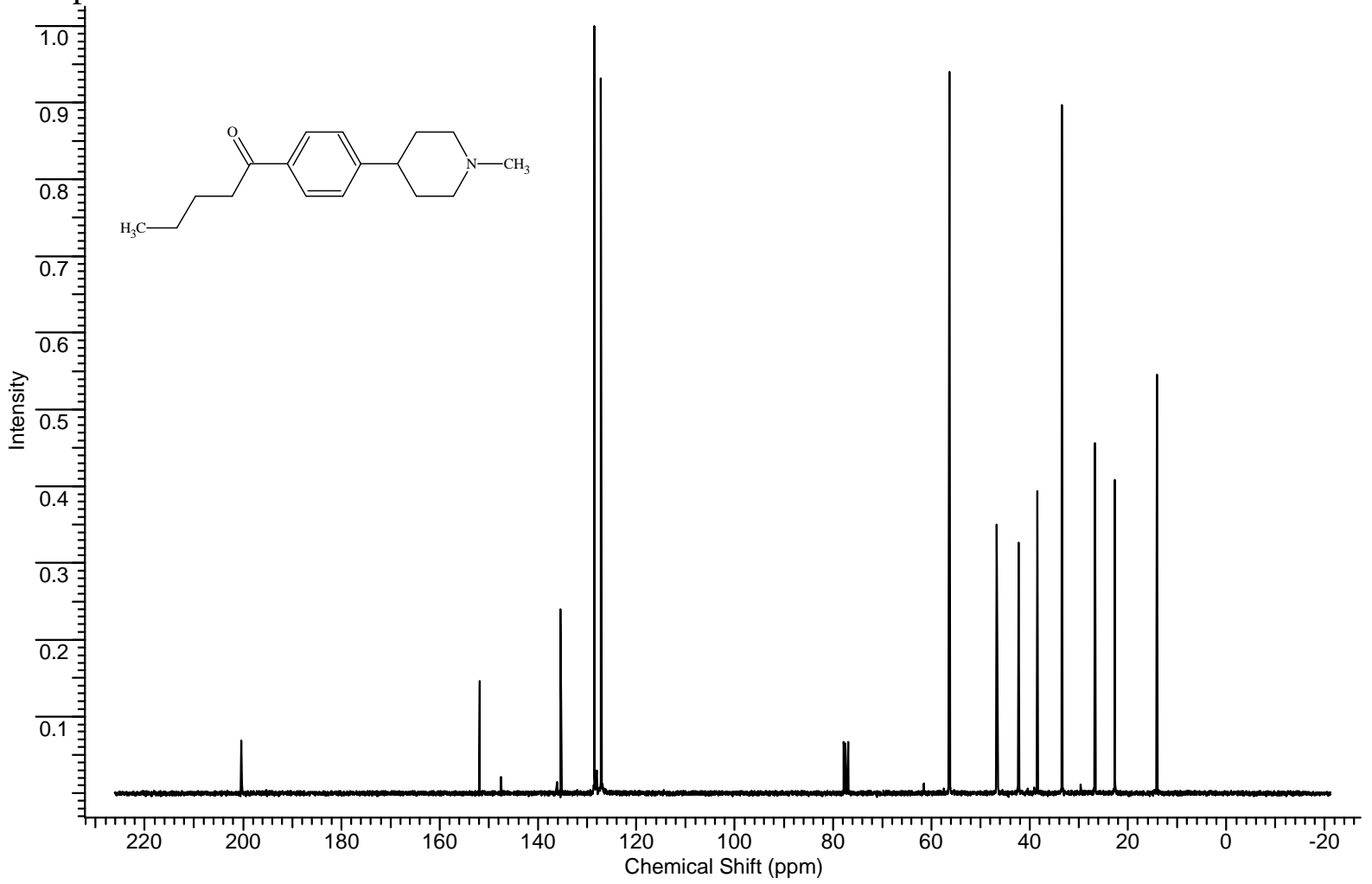




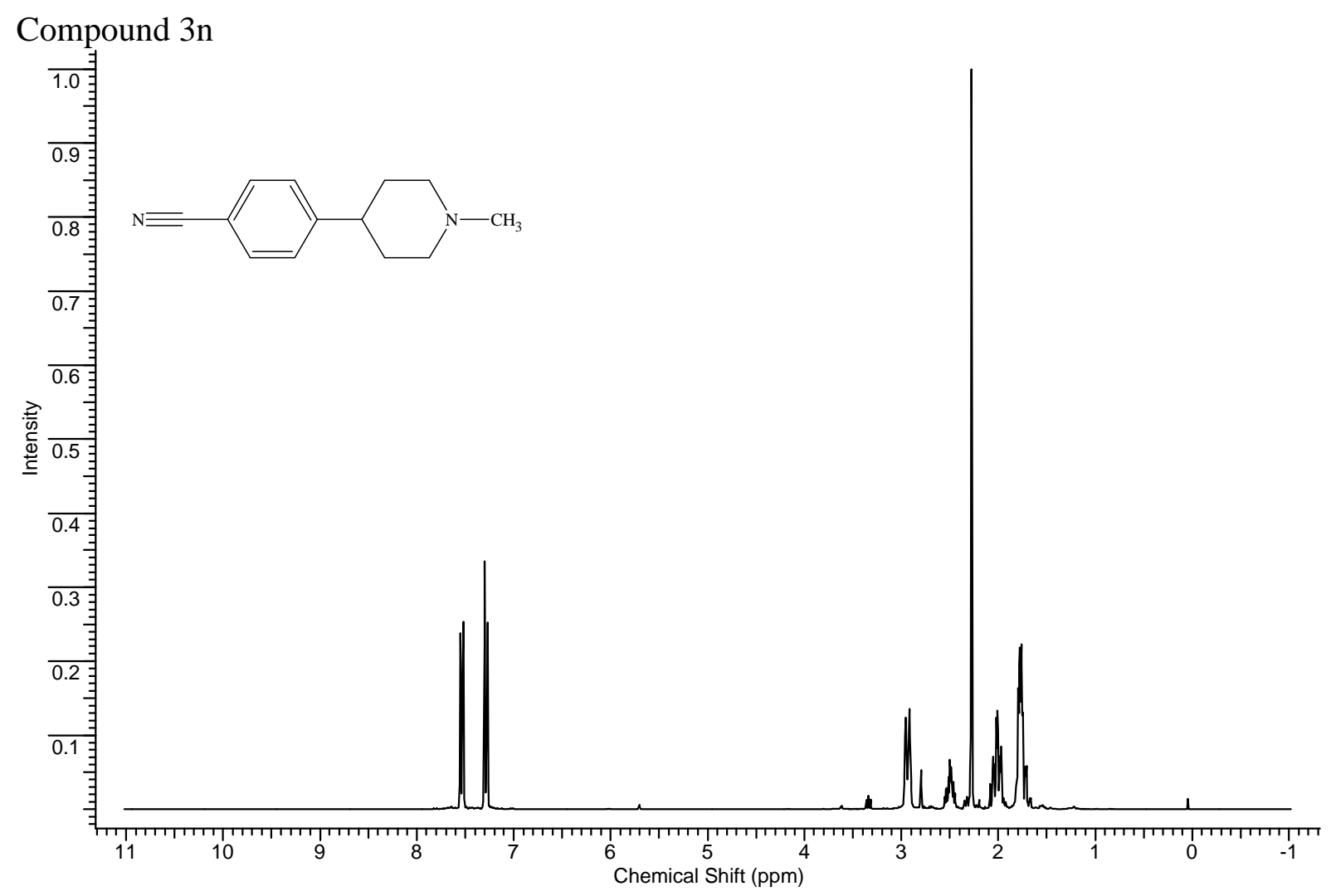

Compound 3n

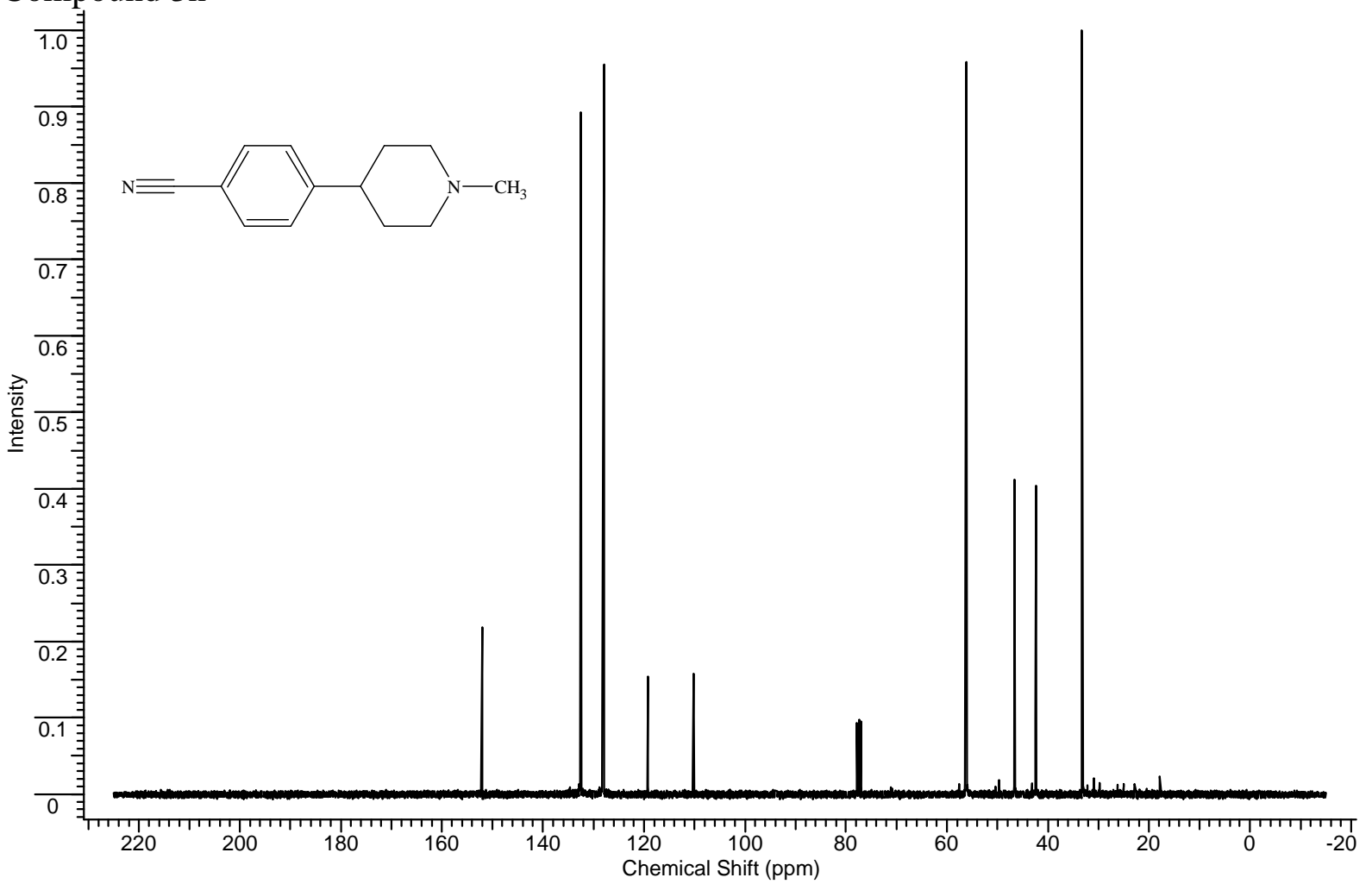




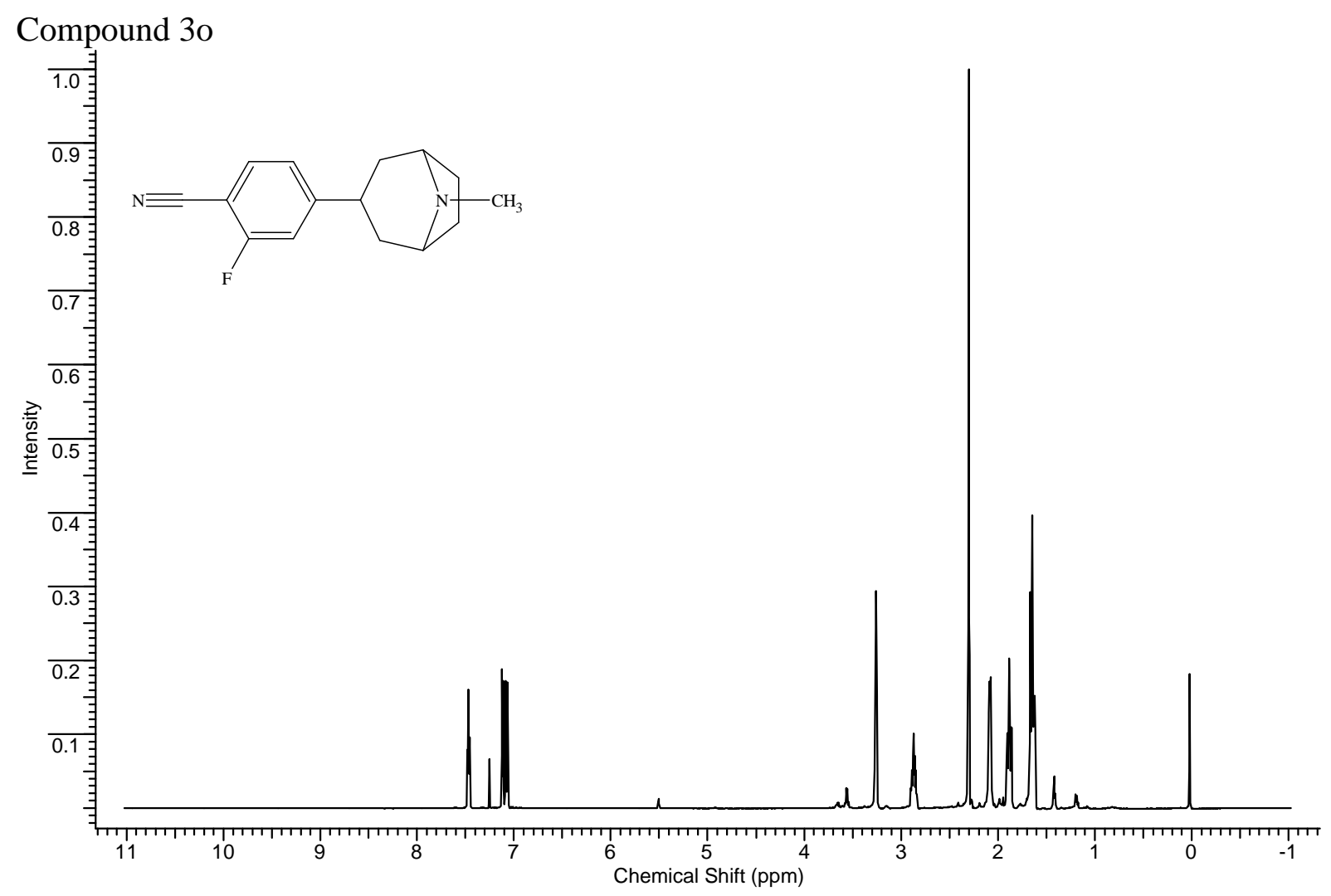

Compound 3o

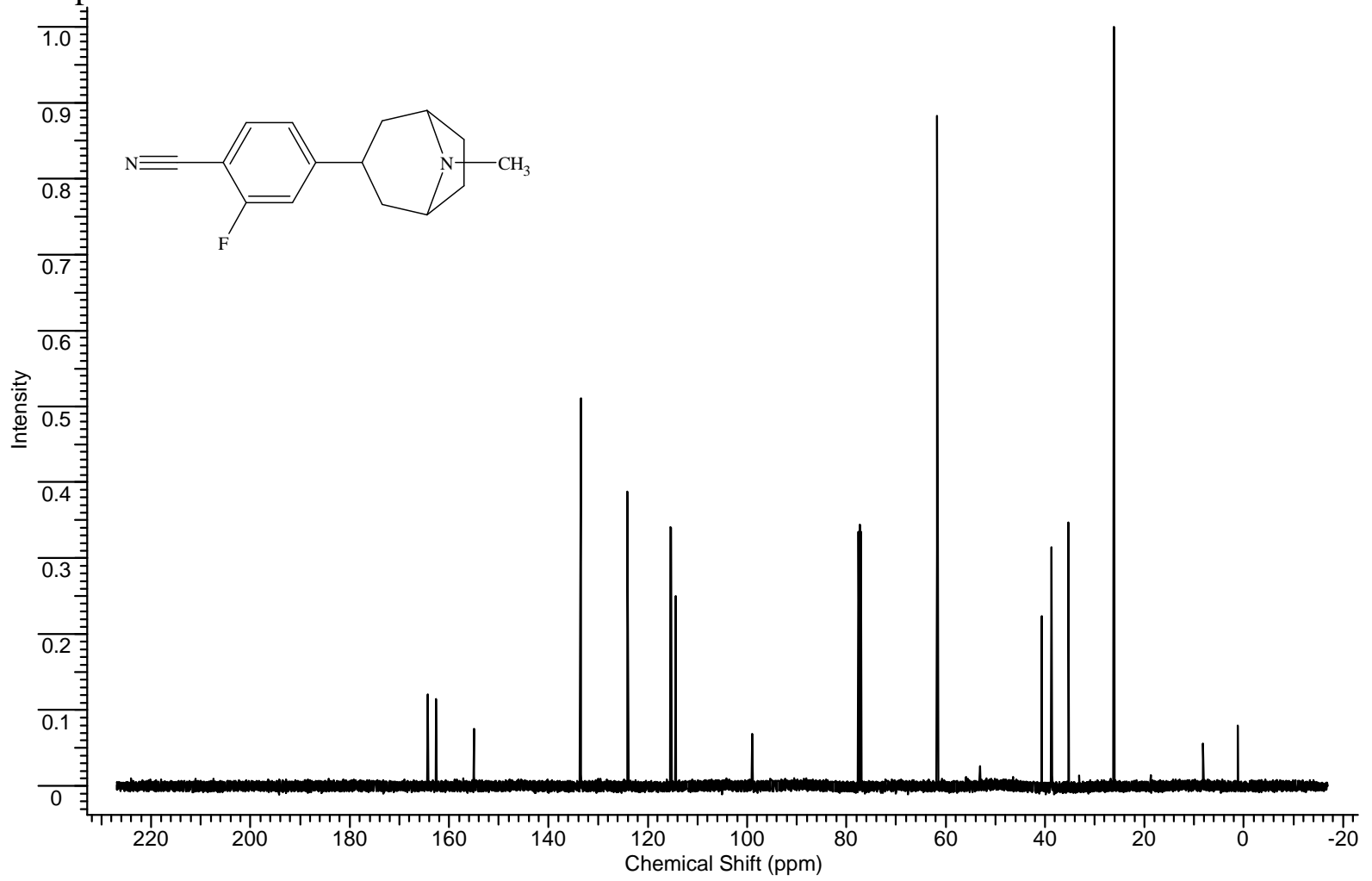



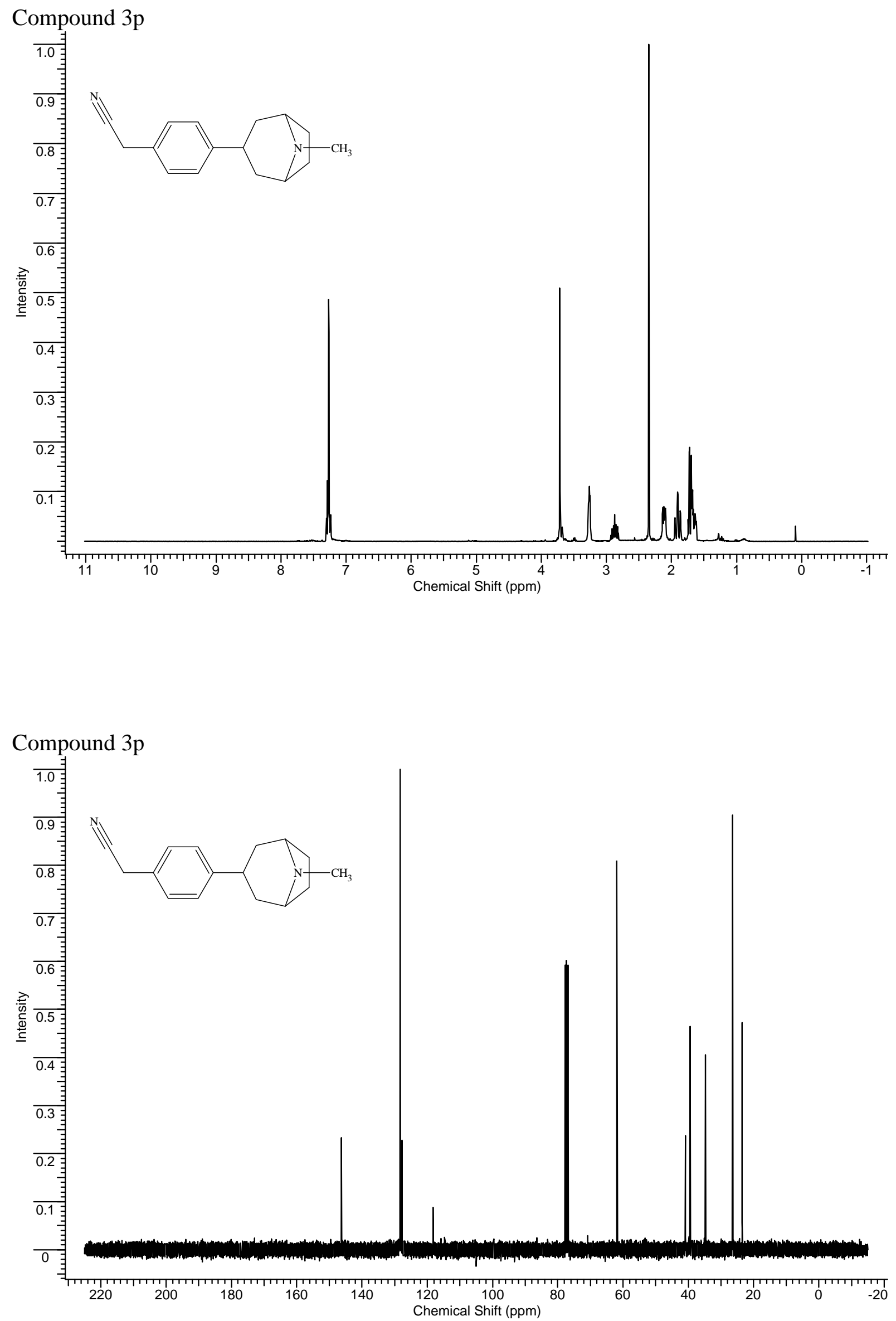

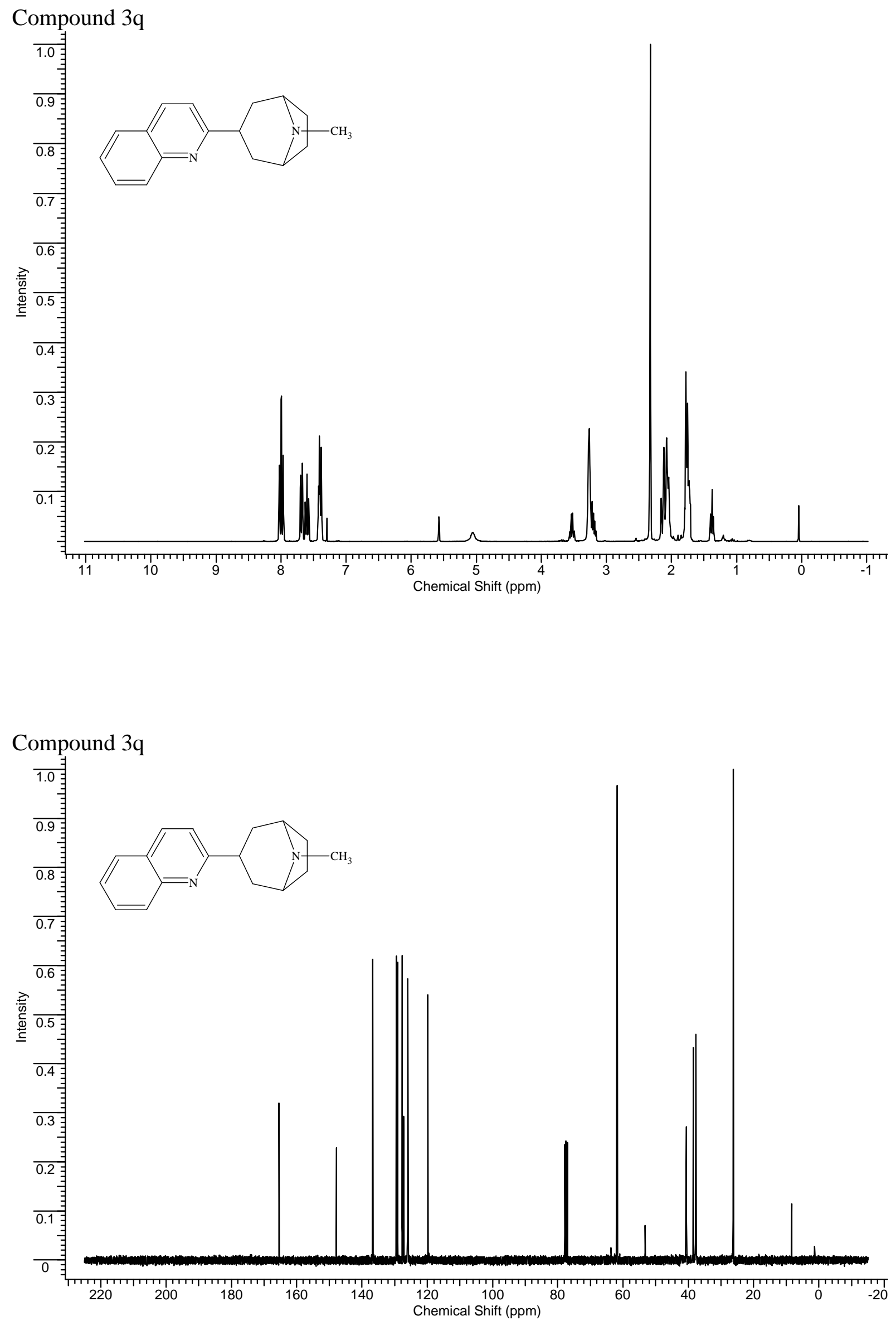